\title{
The Emergence and Evolution of Integrated Worldviews
}

\author{
Liane Gabora \\ Department of Psychology, University of British Columbia \\ Diederik Aerts \\ Center Leo Apostel for Interdisciplinary Studies and Department of Mathematics \\ Free University of Brussels
}

Reference:

Gabora, L. \& Aerts, D. (2009). A model of the emergence and evolution of integrated worldviews. Journal of Mathematical Psychology, 53, 434-451.

Address for correspondence:

Liane Gabora

liane.gabora@ubc.ca

Department of Psychology

University of British Columbia

Okanagan Campus, 3333 University Way

Kelowna BC, V1V 1V7, CANADA 


\section{ABSTRACT}

It is proposed that the ability of humans to flourish in diverse environments and evolve complex cultures reflects the following two underlying cognitive transitions. The transition from the coarse-grained associative memory of Homo habilis to the fine-grained memory of Homo erectus enabled limited representational redescription of perceptually similar episodes, abstraction, and analytic thought, the last of which is modeled as the formation of states and of lattices of properties and contexts for concepts. The transition to the modern mind of Homo sapiens is proposed to have resulted from onset of the capacity to spontaneously and temporarily shift to an associative mode of thought conducive to interaction amongst seemingly disparate concepts, modeled as the forging of conjunctions resulting in states of entanglement. The fruits of associative thought became ingredients for analytic thought, and vice versa. The ratio of associative pathways to concepts surpassed a percolation threshold resulting in the emergence of a self-modifying, integrated internal model of the world, or worldview.

Keywords: cognitive development, concepts, context, cultural evolution, entanglement, evolutionary psychology, conceptual integraton, quantum interaction, SCOP formalism, worldview 


\section{Introduction}

What enabled humans to flourish in diverse environments, and give rise to a process of cultural evolution that is not just cumulative but complex, adaptive, and open-ended? Various hypotheses have been put forward, such as that it was due to onset of language, tool use, or organized hunting. In the paper it is proposed that all of these abilities were made possible through two underlying cognitive transitions.

Like other species, our early ancestors, Homo habilis, could learn, remember, and perhaps even form concepts, but their behavior is much more stereotyped and brittle than our own. Donald (1993) refers to their minds as episodic because they could encode episodes of experience, and were sensitive to their significance, but could not voluntarily access them. The episodic mind can coordinate appropriate actions in response to stimuli, but does not use symbols and makes limited use of concepts, and does not enact events, or refine skills. Therefore its mental life rarely strays from the 'here and now'. Homo erectus appeared approximately 1.55 MYA and may have co-existed with Homo habilis for as long as a half million years (Spoor et al., 2007), but its brain was substantially larger (Bickerton, 1990; Corballis, 1991; Lieberman, 1991). This period is also associated with the origin of culture, as evidenced by such things as the onset of tools, organized hunting strategies, and deliberate use of fire. Donald refers to the mind of Homo erectus as mimetic because it could spontaneously recall events without a cue, and act them out for others. One thing could now stand for or signify another, thus communication took on a semiotic character. It could also forge associations, and refine ideas by modifying internal representations, a process referred to as representational redescription (Karmiloff-Smith, 1990, 1992). Its stream of experience thereby strayed from the 'here and now' into the realm of abstractions.

A second sudden period of encephalization occurred between 500,000 and 200,000 (Aiello, 1996) or 600,000 and 150,000 years ago (Ruff, Trinkaus, \& Holliday, 1997). Some time later, approximately 60,000 years ago, we see the sudden onset of evidence of the creativity characteristic of the modern mind of Homo sapiens, such as task-specific tools, art, jewelry, and signs of ritualized religion (Bar-Yosef, 1994; Klein, 1989; Leakey, 1984; Mellars, 1973, 1989a, b; Mithen, 1996, 1998; Soffer, 1994; Stringer \& Gamble, 1993; White, 1993). The modern mind can form abstract concepts, combine information from different domains (as in analogical reasoning), adapt views and actions to new circumstances, and communicate using the complex syntax and recursive embedding characteristic of modern human languages. It can frame new experiences in terms of previous ones, solve problems using whatever potentially relevant information it can obtain, and formulate plans of action that reflect the specifics of a situation. In short, a modern human behaves as if items in memory are integrated into what we will refer to as a worldview that provide a big picture of what is going on. Its mind is much more than a collection of isolated memories, concepts, attitudes, and so forth; it is a manner of navigating them, weaving narratives with them, and thereby better understanding and interacting with the world. 
However, the existence of this kind of integrated structure presents a paradox. Clearly a worldview takes shape in the course of, not just direct experience, but streams of thought. For example, by contemplating the pros and cons of a democracy one clarifies one's views on democracy. However, streams of thought draw upon abstract concepts and their associations to mediate how one thought gives way to the next. How could one engage in a stream of thought before concepts are woven into an integrated structure, and how could concepts be woven into a integrated structure prior to the capacity for a stream of thought? How could something composed of complex, mutually dependent parts come to be? We have a chicken and egg problem. Moreover, what kind of thing could concepts be given that their properties and meanings shift each time they are called upon? As Bruza et al. (this volume) note, their behavior defies the conditions of classical object-hood and the notion of objectivity.

The scenario we sketch out for how this could have happened is the following. First, we draw upon the notion of autopoiesis as a process that gives rise to structure that is self-organizing, self-preserving, and self-referential (Maturana \& Varela, 1980; Thompson, 2007). Specifically it has been suggested that the chicken and egg problem can be resolved in terms of a process of conceptual integration (Gabora, 1998, 1999, 2000), and in this paper the notion is developed more formally. The idea is inspired by the notion that a major bottleneck to modern cognition was the evolution of self-triggered memory (Donald, 1991). We propose that self-triggered thought is made possible by a memory that is sufficiently fine-grained that episodes are encoded in rich enough detail to regularly evoke associations and remindings. We propose that reminding events induce concept formation, which facilitate chains of associations, since any concept is associatively linked to more than one instance of it, the number of associative paths increases faster than the number of concepts. Graph theory tells us that when this happens, a percolation threshold is reached, at which point the probability rises sharply that they self-organize into a connected closure space (Erdos \& Renyi, 1959, 1960). At this point there exists a possible direct or indirect selftriggered associative pathway starting from any one memory or concept to any other, and a relationally structured worldview thereby emerges. But in order to model this we have to tackle the second problem - the extreme contextuality of concepts once the worldview starts to become integrated. The proposed model grew out of work on the State COntext Property or SCOP theory of concepts, which has been used to model how concepts undergo a change of state when acted upon by a context, and how they combine (Aerts \& Gabora, 2005a, b; Gabora \& Aerts, 2002; Gabora, Rosch \& Aerts, 2008; for work in a similar vein see Bruza \& Cole, 2005; Busemeyer et al. 2006; Busemeyer \& Wang, 2007; Nelson \& McEvoy, 2007; Widdows, 2003; Widdows \& Peters, 2003). The present paper investigates how SCOP can be used to describe more elaborate conceptual integration such as is essential for a coherent worldview. Although the controversy surrounding the issue of modularity is relevant, it is not the focus of this paper. The goal here is to show how conceptual integration can be described drawing upon insights of the application of the quantum formalisms for the modeling of the conceptual aspects of a worldview, noting relevance for 
the theory that the worldview constitutes the basic unit of evolution of culture.

\section{State COntext Property Theory of Concepts}

This section briefly reviews the State COntext Property (SCOP) theory of concepts, which provides the mathematical foundation for the paper. We emphasize that it is a theory of concepts, not words. Thus when we use SCOP to describe how humans began combining concepts, the ordering of the words used to refer to the concepts (e.g. PET FISH) simply reflects the rules of the English language.

SCOP is an elaboration of the State Property formalism (Beltrametti \& Cassinelli, 1981; Aerts, 1982, 1983, 1999, 2002). SCOP explicitly incorporates the context that evokes a concept and the change of state this induces in the concept into the formal description of a concept. With SCOP it is possible to describe situations with any degree of contextuality. In fact, classical and quantum come out as special cases: quantum at the one end of extreme contextuality and classical at the other end of extreme lack of contextuality (Piron, 1976; Aerts, 1983). The rationale for applying it to concepts is expressly that it allows incorporation of context into the model of an entity. ${ }^{1}$

Using the SCOP formalism, a concept is described by five elements:

- A set $\Sigma=\{p, q, \ldots\}$ of states the concept can assume.

- A set $\mathcal{M}=\{e, f, \ldots\}$ of relevant contexts. (Note that contexts can be concepts.)

- A set $\mathcal{L}=\{a, b, \ldots\}$ of relevant properties or features.

- A function $\nu$ that describes the weight (or renormalized applicability) of a certain feature given a specific state. For example, $\nu(p, a)$ is the weight of feature $a$ for the concept in state $p$. Mathematically, $\nu$ is a function from the set $\Sigma \times \mathcal{L}$ to the interval $[0,1]$. We write

$$
\begin{aligned}
\nu: \Sigma \times \mathcal{L} & \rightarrow[0,1] \\
(p, a) & \mapsto \nu(p, a)
\end{aligned}
$$

- A function $\mu$ that describes the transition probability from one state to another under the influence of a particular context. For example, $\mu(q, e$, $p$ ) is the probability that state $p$ under the influence of context $e$ changes to state $q$. Mathematically, $\mu$ is a function from the set $\Sigma \times \mathcal{M} \times \Sigma$ to

\footnotetext{
${ }^{1} \mathrm{SCOP}$ is a general mathematical structure that grew out of (but differs from) the quantum formalism, where the context (which is in this case a measurement) is explicitly incorporated into the theory. Note that this kind of generalization and re-application of a mathematical structure has nothing to do with the notion that phenomena at the quantum level affect cognitive processes.
} 
the interval $[0,1]$, where $\mu(q, e, p)$ is the probability that state $p$ under the influence of context $e$ changes to state $q$. We write:

$$
\begin{aligned}
\mu: \Sigma \times \mathcal{M} \times \Sigma & \rightarrow[0,1] \\
(q, e, p) & \mapsto \mu(q, e, p)
\end{aligned}
$$

Properties in SCOP may be either subsymbolic microfeatures or higher-level features that have a structure of natural relations as described below in section 2.3 (imposed at either the perceptual level or the cognitive level). While in some theories (e.g. Gärdenfors, 2000) context is modeled as a weighting function across attributes or properties, in SCOP any effect of context occurs by way of its effect on the state. ${ }^{2}$ A context may consist of a perceived stimulus or component(s) of the environment, but it is not necessarily the case that the external world figures prominently in the context by which one state gives way to the next. The context may consist entirely of elements of the associative memory or worldview. In other words, the change of state $p \in \Sigma$ to state $q \in \Sigma$ under the influence of context $e$ - hence for which $\mu(q, e, p)$ is the probability - can be internally driven, externally driven, or a mix of both. The value of $\mu(q, e, p)$ depends on such factors as the extent to which $p$ and $q$ are causally related, share properties or conceptual structure at various levels of abstraction, as well as spatiotemporal contiguity of relevant episodes in memory, their relationship to goals and desires, and so forth. Since episodes are understood (and encoded in memory) in terms of the concepts they activate, episodes can be said to have properties and undergo changes of state due to a context just like concepts.

Our minds are able to construct a multitude of imaginary, hypothetical, or counterfactual deviations from the more prototypical states of particular concept, and SCOP can model this. Generally it is not possible to incorporate all of the possible contexts that could influence the state of a concept. The more states and contexts included, the richer the model becomes. The level of refinement is determined by the role the model is expected to play. ${ }^{3}$ What is relevant for our purposes is that unlike other mathematical models of concepts, the potential to include this richness is present in the formalism, i.e., it can incorporate even improbable states, and largely but not completely irrelevant contexts.

\footnotetext{
${ }^{2}$ The inspiration for this choice comes from the physics theories such as quantum mechanics. The primary reason we make this choice is that it is a more ontologically accurate reflection of what a context is and of what a state is. A context has an existence independent of the properties of the concept in question, which is not captured by modeling it as a weighting function across properties.

${ }^{3}$ This is the same methodology as that used to describe the (usually infinite) number of different states for a physical system. The paradox, namely that it is impossible to incorporate all potential contexts into the description, is resolved here as it is in physics. For concrete mathematical models, one limits the description to a well-defined set of the most relevant contexts, hence a corresponding well defined set of states for the entity. But in principle it is possible to refine the model indefinitely, and hence incorporate ever more contexts and states.
} 


\subsection{Ground States}

An important notion in SCOP is the ground state of a concept, denoted $\hat{p}$. This is the 'raw' or 'undisturbed' state of a concept; the state it is in when it is not being evoked or thought about, not participating in the structuring of a conscious experience (such as, most likely, the concept ZEBRA when you began reading this paper). The ground state is the state of being not disturbed at all by the context. One never experiences a concept in its ground state; it is always evoked in some context. The ground state is a theoretical construct; it cannot be observed directly but only indirectly through how the concept interacts with various contexts (which may include other concepts). This is analogous to the fact that a physical system is never in empty space. Just as the idealized notion of a physical system in empty space is the basis of the description of a real physical system in physics, the ground state of a concept plays a fundamental role in the SCOP approach to concepts. The properties that are actual in the ground state are the characteristic or context-independent (Barsalou, 1982) properties of the concept. (The notion of 'ground state' is somewhat similar to the notion of 'prototype'.)

\subsection{Eigenstates, Potentiality States, and Collapse}

We saw how, inspired by how the problem of context is approached in physics, the SCOP approach to concepts incorporates the notion 'state of a concept', i.e. for any concept there exists a potentially infinite number of possible states it can be in depending on the context that elicits it. Consider a concept described by a $\operatorname{SCOP}(\Sigma, \mathcal{M}, \mathcal{L}, \mu, \nu)$. We say that $p \in \Sigma$ is an eigenstate of the context $e \in \mathcal{M}$ iff $\mu(p, e, p)=1$. For example, if state $p$ of the concept CLOTHING is an eigenstate of the context $e$, 'worn to the office' this means that this context $e$ does not cause this particular state to change to another state of CLOTHING. The context can be said to be irrelevant. This would be the case if $p$ is the state OFFICE CLOTHING, and thus the context 'worn to the office' has no effect on it. Note that a state is only an eigenstate with respect to a particular context. Under a different context the same state may not be an eigenstate.

If a state is not an eigenstate with respect to a particular context, then it is a potentiality (superposition-like) state for this context, reflecting its susceptibility to change. For example, given the context 'worn to the office', the ground state of the concept CLOTHING is a potentiality (superposed) state. Indeed the context 'worn to the office' changes this ground state in the state OFFICE CLOTHING.

If $p \in \Sigma$ is a potentiality state of the context $e \in \mathcal{M}$ then $\mu(p, e, p)<1$. Same as with eigenstates, a state is only a potentiality state with respect to a particular context. A potentiality state that is subject to change under the influence of a particular context is not necessarily subject to change under the influence of any other context. Thus it is through the effect of $e$ on $p$ that sensitivity to context is incorporated, and this is what enables a SCOP model 
of a concept to be the rich, imagistic, unbounded mental construct that research has revealed concepts to be. Borrowing terminology from quantum mechanics we refer to this change of state as collapse.

Much as properties of a quantum entity do not have definite values except with respect to a measurement with which they are compatible, properties of a concept do not have definite applicabilities except with respect to a context with which they are compatible. Let us examine how this is dealt with, first in quantum mechanics and then in SCOP.

If a quantum entity prior to a measurement is in an eigenstate of this measurement, then the measurement does not change this state (it just 'detects' what is 'in acto'). If the quantum entity, prior to the measurement, is in a superposition state with respect to this measurement, then the measurement changes this state - collapse - and also changes what is actual and what is potential. Some properties that were actual become potential and vice versa. In the formalism of quantum mechanics, a superposition state is not an absolute type of state in the sense that it is only defined with respect to a measurement. Concretely this means that if a certain measurement is performed, then with respect to this measurement, each possible state of the quantum entity is either a superposition state or an eigenstate. If it is a superposition state, this means that the quantity being measured is potential (i.e. does not have a specific value). If it is an eigenstate, then the quantity being measured is actual (does have a specific value). The effect of the measurement is to change a superposition state to an eigenstate, hence to make quantities that were potential before the measurement actual. Quantities that were actual with respect to another measurement before the considered measurement can become potential with respect to this other measurement after the considered measurement. In other words, a measurement changes superposition states with respect to this measurement into eigenstates, and eigenstates with respect to other measurements into superposition states.

Similarly, if a concept, prior to the context, is in a state of potentiality with respect to this context, then the context changes this state (collapse), and also changes what is actual as well as what is potential. For example, consider the concept TABLE, and two contexts: (1) 'is laid out with food', and (2) 'is cleaned off'. The property 'is laid out with food' is actual for TABLE in a state that is an eigenstate of the first context. In an eigenstate of the second context, as the food is cleared away, this property becomes potential. Another property, 'is cleaned off', which was potential for an eigenstate of TABLE in the first context, becomes actual for an eigenstate in the second context. It is this dynamics of actualities becoming potential and potentialities becoming actual that is described using the quantum formalism. The influence of context on the state of a concept can be such that even characteristic properties of a concept disappear if the concept transforms into a new state under the influence of a context. For example, consider the concept ISLAND. The property 'surrounded by water' is a characteristic property, indeed actual in the ground state of ISLAND. But if we apply the context kitchen to island, and hence consider the concept 
KITCHEN ISLAND, it does not have the property 'surrounded by water' as an actual property (or hopefully not).

\subsection{The Structure of Natural Relations}

Basic level categories (e.g. CAT) mirror the correlational structure of properties as they are perceived, learned, and used by individuals, and further discrimination occurs at the subordinate (e.g. TABBY) and the superordinate (ANIMAL) levels (Rosch, 1978). The structure of this conceptual hierarchy is modeled in SCOP by deriving the structure of natural relations for sets of states $\Sigma$, contexts $\mathcal{M}$, and properties $\mathcal{L}$ of a concept (Aerts \& Gabora, 2005a). Concretely this means the following. If a state $p$ is more constrained than a state $q$ (e.g. TABBY is more constrained than CAT) we say that $p$ is 'stronger than or equal to' $q$, thereby introducing a partial order relation in the set of states $\Sigma$. We denote the relation 'is stronger than or equal to' with the symbol $\leq$. We do the same for the set of contexts $\mathcal{M}$. Thus if a context $e$ is more constrained than a context $f$ (e.g. 'in the water and near a fallen branch' is more constrained than 'in the water') we say $e \leq f$, thereby introducing a partial order relation in $\mathcal{M}$. By saying this is a partial order relation means concretely that it satisfies the following mathematical rules. For arbitrary contexts $e, f, g \in \mathcal{M}$ we assume

$$
\begin{aligned}
\text { reflexivity : } & e \leq e \\
\text { transitivity : } & e \leq f, f \leq g \Rightarrow e \leq g \\
\text { symmetry : } & e \leq f, f \leq e \Rightarrow e=f
\end{aligned}
$$

Next we incorporate more of the complexity of the natural world (and our imperfect internal mirrors of it) into the model by introducing the 'and' context, denoted $\wedge$, and the 'or' context, denoted $\vee$, corresponding to conjunction and disjunction respectively. Thus, given contexts $e$, 'in the water', and $f$, 'near a fallen branch', we can construct the context $e \wedge f$ 'in the water and near a fallen branch'. Similarly, we can construct the context $e \vee f$, 'in the water or near a fallen branch'. Elsewhere it is shown that by adding the 'and' and 'or' contexts, $\mathcal{M}$ obtains the structure of a lattice (Aerts \& Gabora, 2005a; see also Aerts, Aerts \& Gabora, this issue; Widdows \& Peters, 2003).

Next we introduce the 'not' of a context. Given the context $e$, 'in the water', the context 'not in the water' is denoted $e^{\perp}$. Technically we say that through this introduction of the 'not' context, an orthocomplementation structure is derived for $\mathcal{M}$. An orthocomplemented lattice structure is also derived for the set of properties $\mathcal{L}$, making it possible to construct a topological representation of a SCOP in a closure space. (The closure in the closure space appearing naturally at the level of a SCOP of one concept is not straightforwardly linked to the notion of closure that we employ in the theory of an integrated worldview developed in this paper.) 


\subsection{SCOP in Hilbert Space}

The dynamics of concepts and how they are influenced by contexts can be more precisely modeled by embedding SCOP in the complex Hilbert space of quantum mechanics (Aerts \& Gabora, 2005b). This enables the weights of properties to be used to calculate the typicality of exemplars or instances. So for example, a particular outfit may be seen as more typical of one state of CLOTHINGsay, CASUAL CLOTHING - than of another state of CLOTHING - say WORK CLOTHING. But with respect to the present paper, what is most useful about the Hilbert space formulation is that it facilitates the modeling of how states give way or transform into other states. Consider two states $p$ and $q$ of a concept. If these states are not orthogonal, then following the dynamics of state changes implied by Hilbert space, this means that a context $e$ with eigenstate $p$, can undergo a change of state from $p$ to $q$. But even if this is not possible because states $p$ and $q$ are orthogonal it is possible to find another context $f$, such that a collapse from $p$ to $q$ occurs by way of this other context. Concretely, first context $f$ changes $q$ into one of the eigenstates of context $f$, for example $s$. (In Hilbert space $f$ can always be chosen such that $s$ is not orthogonal to $p$.) Then, after context $f$ has done its work, context $e$ changes $s$ to $p$. The overall effect of both contexts $f$ and $e$ is to allow a non-zero probability of changing $q$ to $p$, and this is true for any two states $p$ and $q$. This shows that Hilbert space describes a dynamics of states that are closed, in the sense that one can always be changed into another by applying two contexts one after the other.

When a concept interacts with a context in Hilbert space, it is immediately projected out of the ground state to another state. The change of state induced by a context on a concept is graphically represented in Figure 1 by a vector $x$, a subspace $A$, its orthogonal subspace $A^{\prime}$, and the orthogonal projections $P_{A}(x)$ and $P_{A^{\prime}}(x)$ on both subspaces. The vector $x$ represents the state of a concept, and the subspace $A$ represents a context, while the subspace $A^{\prime}$ represents the negation of this context. Under the context represented by $A$, the state of the concept represented by $x$ collapses to the state represented by the projected vector $P_{A}(x)$, and under the negation of this context the state of the concept collapses to $P_{A^{\prime}}(x)$, which is the orthogonally projected vector $x$.

\subsection{Describing Multiple Concepts with SCOP in Fock Space}

Starting from descriptions of separate concepts in a complex Hilbert space SCOP of standard quantum mechanics, it is possible to generate a description of multiple concepts (Aerts \& Gabora, 2005b, Aerts 2007, 2009). In quantum mechanics the joint entity of multiple entities is described in the Hilbert space that is the tensor product of the Hilbert spaces describing these entities. However, it turns out that the quantum field theory procedure to describe multiple concepts gives rise to a more accurate model of what happens when concepts combine than the tensor product. Fock space is the direct sum of tensor products of Hilbert spaces, so it is also a Hilbert space. It is the fact that it is the direct sum 


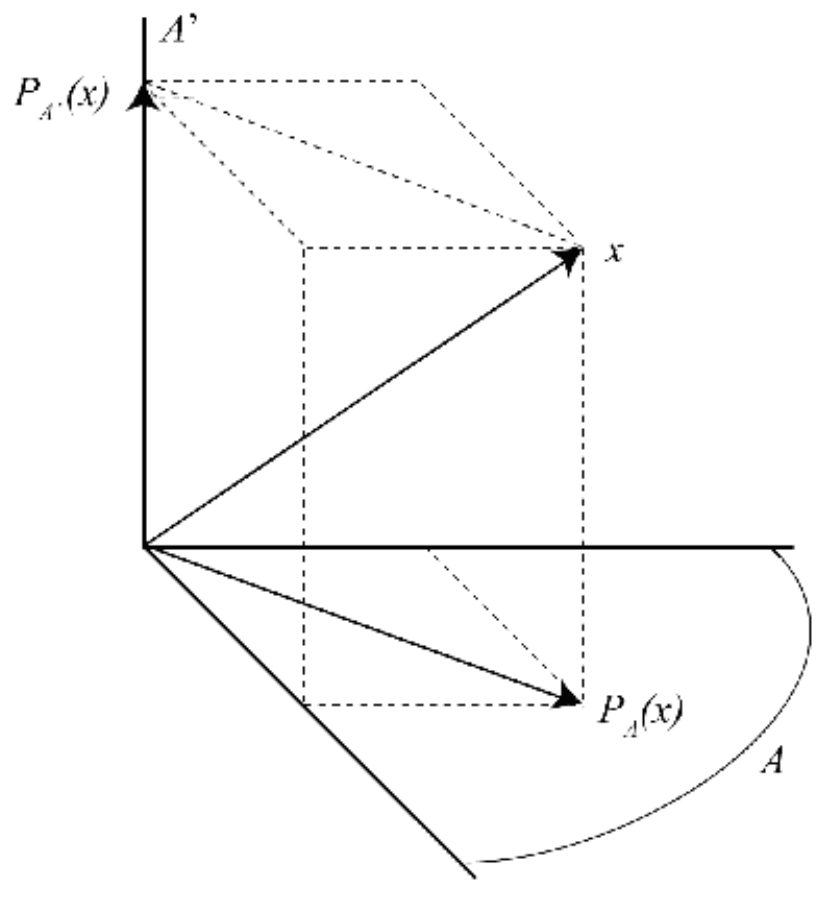

Figure 1: Graphical depiction of a vector $x$ representing the state of a concept, a subspace $A$ representing a context, and its orthogonal subspace $A^{\prime}$ representing the negation of this context. Under the influence of context $A$, state $x$ collapses to the state represented by the orthogonal projection vector $P_{A}(x)$. Under the influence of $A^{\prime}, x$ collapses to the state represented by the orthogonal projection vector $P_{A^{\prime}}(x)$. 
of tensor products of Hilbert spaces that gives Fock space the kind of internal structure that makes it useful for the modeling of multiple concepts and their combinations (Aerts, 2007, 2009). To summarize briefly, suppose we consider a collection of $n$ concepts

$$
\mathcal{U}=\left\{u_{1}, \ldots, u_{j}, \ldots, u_{n}\right\}
$$

and also a combination of these $n$ concepts. First we introduce the complex Hilbert space $\mathcal{H}$ that describes all of these concepts, such that the state of each concept $u_{j}$ is represented in this Hilbert space by mean of a unit vector $\left|u_{j}\right\rangle \in \mathcal{H}$.

Before we model the general situation, let us model the situation that the combination constitutes a new concept. The state $|u\rangle_{\text {new }}$ of this new concept is a weighted linear combination of the states of all the concepts.

$$
|u\rangle_{\text {new }}=\sum_{j=1}^{n} a_{j} e^{i \alpha_{j}}\left|u_{j}\right\rangle
$$

with $0 \leq a_{j} \leq 1$ and $\sum_{j=1}^{n} a_{j}^{2}=1$ and $e^{i \alpha_{j}}$ the phase of each of the complex numbers $a_{j} e^{i \alpha_{j}}$, where we have written the complex numbers of the linear combination in polar form. There is another special situation, namely that the combination is a collection of $n$ distinct and separate concepts, hence the opposite of coming together as 'one' new concept. The state $|u\rangle_{c o l}$ of such a collection of concepts is the tensor product of the states of all concepts.

$$
|u\rangle_{c o l}=\otimes_{j=1}^{n}\left|u_{j}\right\rangle
$$

All the intermediate situations are also possible, of course, e.g. where part of the collection of concepts is treated as a new concept while others remain distinct. Let us mathematically express this general situation, hence also including the intermediate ones. First we write the state of the situation where for $0 \leq k \leq n$, there are $k$ subsets $\left\{\mathcal{U}_{j} \mid j=1 \ldots k\right\}$ of $\mathcal{U}$, hence $\mathcal{U}_{j} \subseteq \mathcal{U} \forall j$ and $\mathcal{U}=\cup_{j=1}^{k} \mathcal{U}_{j}$ and $\mathcal{U}_{j_{1}} \cap \mathcal{U}_{j_{2}}=\emptyset$ for $j_{1} \neq j_{2}$, such that for each of these subsets there is a new concept. The state of this concept is then the weighted linear combination of the states contained in that subset. Since we have considered $k$ such subsets, the global state of all of the collection of concepts is then a vector of $\otimes_{j=1}^{k} \mathcal{H}_{j}$, the $k$-times tensor product of the Hilbert space $\mathcal{H}$. This means that the state of the collection is then the weighted superposition of each of these states, which is an element of the direct sum for all values of $k$ of $\otimes_{j=1}^{k} \mathcal{H}_{j}$, hence of the space $\oplus_{k=1}^{n} \otimes_{j=1}^{k} \mathcal{H}_{j}$, which is Fock space. A general state $|u\rangle_{\text {gen }}$ of this Fock space can be written as

$$
|u\rangle_{g e n}=\frac{1}{N} \sum_{k=1}^{n} m_{k} e^{i \theta_{k}}\left(\sum_{l} a_{l k} e^{i \alpha_{l k}}\left(\otimes_{j=1}^{k}\left|v_{j l}\right\rangle\right)\right)
$$

where $N$ is a normalization constant such that $\left\langle u_{\text {gen }} \mid u_{\text {gen }}\right\rangle=1$, and $\left|v_{j l}\right\rangle \in \mathcal{H}$ are states of the individual concepts, however not necessarily of the forms $\left|u_{j}\right\rangle$. Some of them might be of this form; others might be weighted superpositions of 
such states, if they express combinations of parts as a whole, and others might be orthogonal projections of such states, if the effects of contexts are taken in account explicitly in a combination. The sum $\sum_{l} a_{l k} e^{i \alpha_{l k}}\left(\otimes_{j=1}^{k}\left|v_{j l}\right\rangle\right)$ expresses that we consider also entangled states of such $k$ tensor product states $\otimes_{j=1}^{k}\left|v_{j l}\right\rangle$ as possible states of that part of the general state of the combination of concepts described in sector $k$ of Fock space.

Consider an example, namely the conception of a child playing in the garden. The individual concepts are CHILD, PLAY, and GARDEN. Each of them is described by a ket-vector, hence we have the set of vectors $\mathcal{U}=\left\{\left|u_{1}\right\rangle,\left|u_{2}\right\rangle,\left|u_{3}\right\rangle\right\}$, where $\left|u_{1}\right\rangle$ is the state of CHILD, $\left|u_{2}\right\rangle$ is the state of PLAY, $\left|u_{3}\right\rangle$ is the state of GARDEN. The state denoted $|u\rangle_{\text {new }}$ is the weighted superposition of the three states that represent the combination as one new concept. The state denoted $|u\rangle_{\text {col }}$, is the 3 tensor product state of the 3 states. It represents them as a collection of separate concepts. The intermediate situations are those for which subsets of the collection of concepts are considered new concepts, while these subsets themselves are considered to constitute distinct elements of the whole set. All these situations are described by the state denoted $|u\rangle_{g e n}$ of Fock space.

Many subtle aspects of how concepts come together can be modeled by the Fock space representation. If two concepts come together as a new conceptual element, then in the Fock space state representation, the linear combination coefficient giving rise to the weight for this factor becomes dominant compared to those for other concepts that do not come together to be considered as something new. There is a dynamical shifting of the weights in Fock space. Thus the quantum modeling scheme contains the fine structure to describe the variety of ways one could react to a particular collection of concepts. This is why we model a cognitive state as the state of Fock space that describes all concepts and combination of concepts of the worldview at a particular instant.

One additional remark is at place here. The Fock space procedure makes it possible to construct mathematically the state of a combination of many concepts. However, some combinations are more easily modeled using a procedure described detail elsewhere (Aerts \& Gabora 2005a,b). These are the combinations where certain concepts are brought into the conceptual structure as contexts rather than as entities. We have incorporated concepts that act as a context in a combination in the Fock space representation, since in equation (9) we suppose the considered collection $\mathcal{U}$ to consists of concepts that are entities in the considered combination, hence not concepts that are contexts. However, we allow concepts that are context to change the states of the entity concepts as described above, and this possibility is contained in equation (9). The description put forward here is also fine enough to take into account that the distinction between a concept playing the role of context or rather playing the role of entity within a specific combination can be ambiguous, and can also change depending on the overall context. A good example is STONE LION. Different possible ways of looking at this combination exist, and a positive answer to both questions 'is STONE LION a LION' and 'is STONE LION a STONE' make sense. Hence a better approach is to consider both as entities, and use Fock space for 
a two entity situation, and forgo the more simple model of considering one of them as a context.

\section{SCOP Applied to Cognitive States}

Until now SCOP has been used to describe one or more concepts. This section presents material needed to apply it to the entire set of concepts possessed by an individual, as is necessary to model the evolution of an integrated worldview. We first discuss the structure of associative memory, and the distinction between states of a concept and cognitive states, and outline the various kinds of internal pathways involved in the transformation or collapse from cognitive state to another.

\subsection{Ways of Incorporating Associative Structure in Mod- els of Cognition}

It is well-known that memory neurons respond to specific properties, or subsymbolic microfeatures (Churchland \& Sejnowski, 1992; Smolensky, 1988). For example, one might respond to a particular shade of blue, or the quality of 'stickiness', or quite likely, something that does not exactly match an established term (Mikkulainen, 1997). Items are distributed across a cell assembly that contains many neurons, and likewise, each neuron participates in the storage of many items. Thus, the same neurons get used and re-used in different capacities, a phenomenon referred to as neural re-entrance (Edelman, 1993). Items stored in overlapping regions are correlated, or share features. Memory is also content addressable, i.e. there is a relationship between the semantic content or meaning of an item, and which neurons are activated by it and participating in encoding it. As a result, episodes stored in memory can thereafter be evoked by stimuli that are similar or resonante in some (perhaps context-specific) way (Hebb, 1949; Marr, 1969). It follows that the likelihood that one item evokes another varies with the extent to which they share properties. Most models of concepts include only a small number of properties as possible properties for a concept to have, which inevitably is too limited to account for what happens when a concept appears in a context quite different from previous ones, or when it combines with other concepts. As mentioned previously, SCOP allows that for each concept there exists some context that could actualize a certain property that was thought to be very unlikely for that concept; thus each of the properties can potentially play a possible role in any concept (though the weights for most properties for any given state will tend to equal zero).

Kanerva (1988) makes some astute observations about the conceptual space that can be realized by a sparse, distributed, content-addressable memory such as human memory. We denote the number of subsymbolic microfeatures, or properties, that are activated by some neuron and can be encoded in the memory as $n$. The set of all possible $n$-dimensional items that can be encoded in the memory are represented as the set of vertices (if features assume only binary 
values) or points (if features assume continuous values) in an $n$-dimensional hypercube, where the $s$ items that actually are encoded in the memory occupy some subset of these points. The distance $d$ between two points in this space is a measure of how dissimilar they are, referred to as the Hamming distance. The number of items encoded in memory at Hamming distance $d$ away from any given item is equal to the binomial coefficient of $n$ and $d$, which is well approximated by a Gaussian distribution (Figure 2). Thus, if item $\mathrm{X}$ is $111 \ldots 1$ and its antipode is $000 \ldots 0$, and we consider $\mathrm{X}$ and its antipode to be the 'poles' of the hypersphere, then approximately $68 \%$ of the other items lie within one standard deviation $(\sqrt{n})$ of the 'equator' region between these two extremes. As we move through Hamming space away from the equator toward either $\mathrm{X}$ or its antipode, the probability of an item falls off sharply by the proportion $\sqrt{n} / n$.

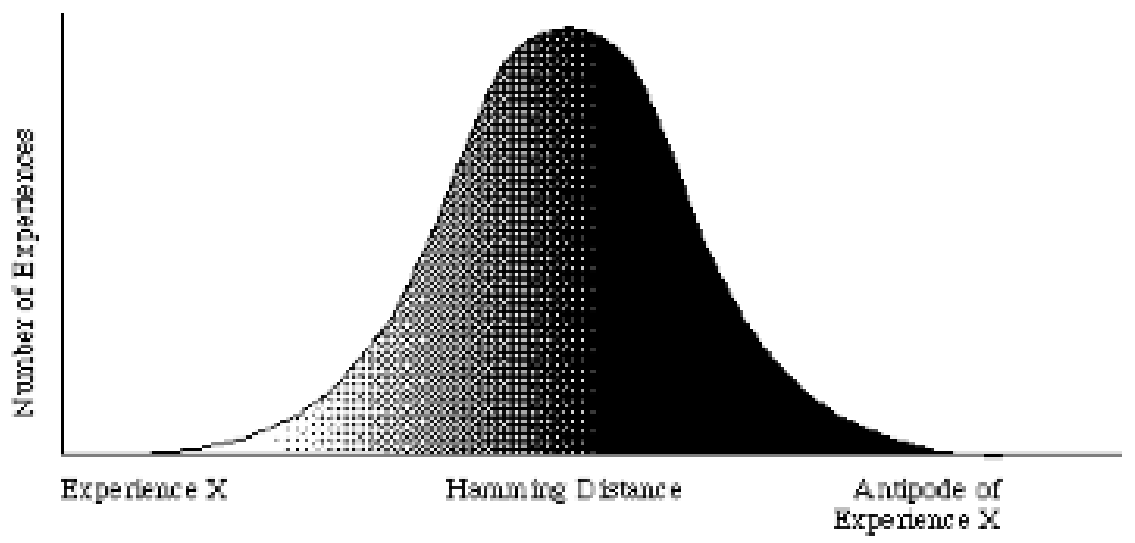

Figure 2. Solid black curve is a schematic distribution of the Hamming distances from any given item to other items encoded in memory. The Gaussian distribution arises because there are many more ways of sharing an intermediate number of features than there are of being extremely similar or different. Activation decreases with distance from the most similar address, as indicated by green shading. (A typical computer memory stores each item in only the left-most address, whereas a distributed network stores it throughout the network. A neural network that uses an activation function, such as the radial basis function, is intermediate between these two extremes.)

Of course conceptual distance is context sensitive, but not in a chaotic way; thus for example, ROSEBUD is closer to PANSY than to SPACESHIP across the vast majority of contexts. So the Hamming distances here are for items in their most prototypical forms, i.e., their most typical or commonly encountered contexts.)

Note that Figure 2 depicts not the distribution of actual items in memory, but of possible items; with respect to actual items encoded in memory, the tail regions would be considerably sparser. This means that while there are many ways of being somewhat similar to any given item, very few items are 
either very similar to, or very dissimilar to, a given item, and the lower $n$ is the more pronounced the effect. This has implications for the model developed here because interaction and integration is more probable amongst items that are similar (or more correctly, more correlated). Langton's (1992) finding that the information-carrying capacity of a system is maximized when its interconnectedness falls within a narrow regime between order and chaos suggests that to produce a steady stream of meaningfully related yet potentially creative remindings, the correlation between one thought and the next must fall within a narrow range.

It is interesting to look at how characteristics of associative memory are realized by SCOP as compared to a neural network. A neural network is contextsensitive in the sense that the output depends on (1) not just one feature but all features of an input, (2) what patterns the neural network has learned previously (reflected in the weights on links between nodes), and (3) the activation function that gives the relationship between activation of a node and its output to other nodes. The fact that items are distributed in memory is modeled in a neural network through parallel distributed activation of nodes. The fact that memory exhibits coarse-coding, such that a given neuron responds maximally to one microfeature but responds to a lesser degree to other similar microfeatures, can be modeled by constraining the distribution using a radial basis function (RBF). Each input activates a hypersphere of hidden nodes, with activation tapering off in all directions according to a (usually) Gaussian distribution of with standard deviation $\sigma$ (Willshaw \& Dayan, 1990; Hancock et al., 1991; Holden \& Niranjan, 1997; Lu et al., 1997). This enables one part of the network to be modified without interfering with the capacity of other parts to store other patterns. Where $F$ is the activation, $x$ is an i-dimensional input vector, and $k$ is the center of the $\mathrm{RBF}$, hidden nodes are activated as follows

$$
F(x)=e^{-\sum_{i}\left(\left(x_{i}-k_{i} / \sigma\right)^{2}\right)}
$$

The further a node is from $k$, the less activation it receives from the input and in turn contributes to the output. If $\sigma$ is small, the input activates few neurons but these few are hit hard; we say the activation function is spikey. If $\sigma$ is large, the input activates many neurons to an almost equal degree; we say the activation function is relatively flat. In effect $\sigma$ moderates the degree to which the output is affected by other patterns that have previously been encoded.

This approach nicely resolves the problem of interference amongst stored patterns, but like all neural net approaches, it assumes the validity of the notion of spreading activation, which has recently been seriously called into question (Bruza, Kitto, Nelson \& McEvoy, this volume; Nelson, McEvoy \& Pointer, 2003; Nelson \& McEvoy, 2007). In SCOP, a similar effect can be obtained using a variable we will refer to as the association threshold, denoted $\phi$, which adjusts the extent to which associations constitute part of the context that evokes the change of state from $p$ to $q$. For example, at a given moment you may be pruning a tree. Let us refer to this cognitive state as p. In your next cognitive state, let's call it q, you might be reminded of another tree you have pruned. 
This change of cognitive state is possible because both $\mathbf{p}$ and $\mathbf{q}$ are instances of the concept TREE-PRUNING with a non-zero transition probability $\mu(q, e$, $p$ ), The change of cognitive state is the result of associative recall (as discussed in section 3.2.2), i.e., there exists a pathway between $\mathbf{p}$ and $\mathbf{q}$ denoted $\mathbf{p} \rightarrow \mathbf{q}$.

Alternatively your associations may be not at the level of properties but at the conceptual level; you might be reminded of your family tree, or the tree of knowledge. You might be hungry or your hand might be getting sore. All of these are possible components of the context that evokes the collapse from state $p$ of the concept to state $q$. The function $\phi$ modulates how you draw upon these various elements of your situation to create a specific context by which your current thought transforms into your next thought, as follows. If $\phi$ is low, how $p$ transforms into $q$ is affected by other episodes previously encoded in the neurons activated by $p$. The context that evokes the collapse is composed of associations at the property level and/or at other higher levels of abstraction. In other words, we posit that the direction of one's thought is shaped by a choice of contexts that takes associations into account. Since it is impossible to model with complete precision how these associations affect the collapse from $p$ to $q$ the model contains a lack of knowledge and $\mu$ enables us to incorporate this lack of knowledge into the model. Lowering $\phi$ allows increasingly distant associations to be made; referring to figure 2 it will tend to increase how great the Hamming distance can be (how far into the grey area) between the current experience (experience X) and an item in memory in order for that item to be evoked by the current experience. If on the other hand $\phi$ is high, associations play little role; in other words, how $p$ transforms into $q$ is not affected by what other episodes have been encoded in the neurons activated by $p$. Since mental energy is not going toward finding associations it is freed up for symbolic operations on $p$. Since associations do not affect the collapse from $p$ to $q$ there is much less lack of knowledge, hence $\mu$ values tend to be 0 or 1 .

\subsection{Cognitive States versus States of a Concept}

A cognitive state of the mind of an individual is a state of the composition of all of concepts and combinations of concepts of the worldview of this individual. Referring to the mathematical representation for a large combination of concepts that we put forward in section 2.5, we can consider the state representing the combination of all concepts contained in the worldview of an individual, i.e., the cognitive state of the mind of this individual. Hence, making use of equation (9), and denoting by $\mathcal{U}$ the collection of concepts available to an individual, where we denote the state of an concept $u \in \mathcal{U}$ by means of the ket-vector $|u\rangle \in \mathcal{H}$, where $\mathcal{H}$ is a complex Hilbert space, this gives

$$
\mathbf{p}=\frac{1}{N} \sum_{k=1}^{n} m_{k} e^{i \theta_{k}}\left(\sum_{l} a_{l k} e^{i \alpha_{l k}}\left(\otimes_{j=1}^{k}\left|v_{j l}\right\rangle\right)\right)
$$

where $N$ is a normalization constant such that $\langle\mathbf{p} \mid \mathbf{p}\rangle=1$, and $\left|v_{j l}\right\rangle \in \mathcal{H}$ represent states of the individual concepts, however not necessarily of the forms $|u\rangle$ 
for an $u \in \mathcal{U}$. Some of them might be of this form, others might be weighted superpositions of such vectors, and still others might be normalized projections of such vector due to concepts that only function as context in the considered combination (we have not included such concepts in $\mathcal{U}$ ). For a given cognitive state $\mathbf{p}$, most concepts $u \in \mathcal{U}$ are in their ground state. They are not evoked in this instance of experience, hence we say they are not activated. Those concepts $u \in \mathcal{U}$ that are not in their ground states for the cognitive state can be said to be activated, or in the language of quantum mechanics we say they are in 'excited states'.

Note that $\mathcal{U}$ refers to the totality of what has been conceived (framed in conceptual terms), not what has been perceived. It is possible that one could have an experience that activates memory but such that it is not framed in conceptual terms. For example, one might have an experience (such as "I like the white pebble in the water") without verbalizing it as such even to oneself, or in the case of an infant without even possessing the concepts WHITE, PEBBLE, and WATER. That is, one might simply look at the thing and like it. We reserve the term cognitive states to refer specifically to episodes that activate concepts. A cognitive state that is not conceptualized, i.e. not interpreted in terms of concepts, may still give way to another state through cognitive events such as reminding, induction, and so forth, but this change of state reflects the set of properties $\{a, b, \ldots\}$ of the current episode, and the properties of similar previously encoded episodes, not the conceptual structure by which these sets of properties have been interpreted and organized.

We use the term episode to refer to a cognitive state that is either being encoded or has been encoded in long-term memory and that can thus potentially be recalled from memory (either completely or partially, accurately or inaccurately). The content of an episode can range from rich perceptual data to a highly abstract or imaginary mental construct that has little relation to something ever directly perceived. The collection of all episodes is $\mathcal{X}$, and episodes will be denoted $x, y, z \in \mathcal{X}$.

Change of cognitive state is recursive. The new cognitive state is a function of the effect on the worldview of (1) the previous cognitive state and (2) the perceived world. Because we use the quantum formalism, or its generalizations, to mathematically describe states, contexts, and properties, it follows from this quantum formalism how dynamics should be modeled. One models dynamics by building the Schrödinger equation, or the generalized version of it. The Schrödinger dynamics models the change that is provoked by the influence of all the contexts. Mathematically we write

$$
i \frac{\partial}{\partial t} \mathbf{p}(t)=H \mathbf{p}(t)
$$

where $H$ is the Hamiltonian, which is a self-adjoint operator in the Hilbert space $\mathcal{H}$. Let us write the exponential form of the Schrödinger equation, which is equivalent with the standard form, but more easy to interpret in the situation 
where we use it. We have

$$
\mathbf{p}(t)=e^{i H\left(t-t_{0}\right)} \mathbf{p}\left(t_{0}\right)
$$

This means that the cognitive state at an arbitrary time $t$ is given by the cognitive state of time $t_{0}$, transformed under influence of the unitary transformation which is the exponential of $i$ times the Hamiltonian operator multiplied by the time interval $t-t_{0}$.

When quantum mechanics is applied in physics to describe the the microworld, the Hamiltonian $H$ of a quantum entity under study is the operator corresponding to the energy of the entity. Hence, it is the energy of the quantum entity that determines how its state changes under the joint influence of all contexts. There is no obvious way to define a notion that plays the role of 'energy' in our situation where the entity is a worldview instead of a quantum entity. One possibility is to approach this situation the other way around. One looks for a self-adjoint operator $H$, such that the dynamics of a worldview is described well by the Schrödinger equation, and then the quantity corresponding to this self-adjoint operator is introduced as being the energy of the worldview. ${ }^{4}$

\subsection{Ways of Forging Conceptual Structure}

This section identifies processes through which conceptual structure is formed. This includes the formation of concepts, and of pathways connecting episodes to episodes, episodes to concepts, and concepts to other concepts. In other words, pathways provide internally generated means of going from thinking about or experiencing one thing to thinking about or experiencing another. The strengths of these pathways is reflected in the transition probabilities of SCOP.

\subsubsection{Pavlovian Conditioning and Spatiotemporal Contiguity}

One source of pathways arises through Pavlovian or classical conditioning, as in the pairing of a bell with food. The episode (bell with food) can be said to instantiate multiple concepts (BELL and FOOD). At the heart of classical conditioning is spatiotemporal contiguity-the pairing of a conditioned stimulus with an unconditioned stimulus causes a response-an association-to the unconditioned stimulus. However neutral stimuli that go together in space and /or time can similarly become associated. To say that two or more properties are properties of a particular concept or cognitive state is to say that they possess associative strucure due to spatiotemporal contiguity, thus this kind of structure is built into SCOP. For example, whether or not BELL has been paired to FOOD, most instances of BELL involve the sight and sound of a bell-two

\footnotetext{
${ }^{4}$ The Schrödinger equation has been used before in this way to model the dynamics of a cognitive entity. Aerts, Broekaert and Smets (1999a,b) use Schrödinger equations to model the cognitive dynamics of the Liar paradox. Busemeyer, Matthew and Wang (2006), use the Schrödinger equation in a quantum game theoretic setting to model a decision of the type where the disjunction effect appears, and a Hamiltonian is calculated with respect to experimental data measured in this situation.
} 
dissimilar properties. As another example the episode 'Mary is wearing a ball gown' is an instance of the concept MARY denoted $u \in \mathcal{U}$ and an instance of the concept BALL GOWN denoted $v \in \mathcal{U}$. Following the experience of this episode, thoughts of Mary could remember one of ball gowns and thoughts of ball gowns could remind one of Mary. We say that there exists a bidirectional pathway between $u$ and $v$ denoted $u \leftrightarrow v$.

\subsubsection{Associative Recall}

Another source of pathways arises through the experience of an episode that is so similar to some previously encoded episode that it evokes a reminding or recall of that previous episode. For example, the episode 'Mary is wearing a ball gown' may evoke a reminding of the episode 'Jane is wearing a ball gown'. Let $\mathbf{p}$ be the cognitive state of experiencing the episode 'Mary is wearing a ball gown' and $\mathbf{q}$ be the cognitive state of experiencing the episode 'Jane is wearing a ball gown'. If $\mathbf{p}$ evokes $\mathbf{q}$ we say that there exists a pathway between $\mathbf{p}$ and $\mathbf{q}$ denoted $\mathbf{p} \rightarrow \mathbf{q}$.

\subsubsection{Abstraction}

An important source of conceptual structure is recognition that particular episodes could be seen as states of a new concept, resulting in the formation of that new concept through the process of abstraction. This can occur when due to the distributed nature of memory, the associative recall process retrieves an episode with substantial overlap of features or conceptual structure, An example is CAT reminds one of DOG resulting in formation of the concept PET. Abstraction generally involves the formation of a more abstract concept out of less abstract (more constrained) concepts. With SCOP this is described as follows. Suppose that $p$ is an episode involving APPLE (and perhaps another episode $q$ involving ORANGE). Abstraction occurs if one realizes (or is told) that there could exist a concept $u \in \mathcal{U}$, the concept FRUIT represented by the SCOP $\left(\Sigma_{u}, \mathcal{M}_{u}, \mathcal{L}_{u}, \mu_{u}, \nu_{u}\right)$ such that $p \in \Sigma_{u}$, and similarly $q \in \Sigma_{u}$. This can arise spontaneously as a result of associative recall; for example an apple reminds one of an orange which leads to the formation of an item in memory that encompasses both.

\subsubsection{Identifying Natural Relations}

Pathways are forged through the learning or recognition of natural relations as discussed in section 2.3. For example consider the concepts $p$ and $q$. If one learns or realizes that $e \wedge f \leq e$, a pathway $e \wedge f \leftrightarrow e$ is generated. Similarly if one learns or realizes that $e \leq e \vee f$, a pathway $e \leftrightarrow e \vee f$ is generated.

An important kind of pathway is the identification of episodes as states or instances of a particular concept, or of concepts as states of more abstract concepts. This is referred to as instantiation. With SCOP this is described as 
follows. Suppose that $u \in \mathcal{U}$ is the concept APPLE, represented by the SCOP $\left(\Sigma_{u}, \mathcal{M}_{u}, \mathcal{L}_{u}, \mu_{u}, \nu_{u}\right)$, and $p$ is a state of apple, say, FUJI APPLE. If one learns or recognizes that $p \in \Sigma_{u}$, then we say that there is an 'isa' pathway connecting $p$ with $u$, denoted $p \rightarrow u$, as well as a pathway $u \rightarrow p$.

If there exists a concept $w \in \mathcal{U}$ such that $p$ and $q$ are both known to be states or instances of $w$, hence $p, q \in \Sigma_{w}$. Thus $p \leftrightarrow w$ and $q \leftrightarrow w$. Since $p \rightarrow w$ and $w \rightarrow q$, through transitivity, $p \rightarrow q$. In such cases we refer to $p$ and $q$ as sister instances of $w$.

\subsubsection{Conjunctions}

It is not always the case that a new concept is more abstract or less constrained than the concepts that gave rise to it. Another important source of conceptual structure is the formation of a conjunction of concepts, as in the joining of the concepts SNOW and MAN to give the idea of a man built out of snow, a SNOWMAN. This occurs when due to the distributed nature of memory, what the associative recall process retrieves has components that lack substantial overlap of features or properties, and that under most contexts would not be retrieved together, and were thought to be unrelated. In SCOP this is mathematically described as in Section 2.5. Two concepts combine to give a conjunction using the tensor product formulation which results in (amongst other states) states of entanglement. Consider a joint concept $u_{1} \otimes u_{2}$ described in the complex Hilbert space SCOP formed by means of the tensorproduct $\mathcal{H}_{1} \otimes \mathcal{H}_{2}$ of the two Hilbert spaces $\mathcal{H}_{1}$ and $\mathcal{H}_{2}$. If one or more of the states $p \in u_{1} \otimes u_{2}$ is an entangled state, we say that there exists a bidirectional pathway connecting the two concepts that comprise the conjunction, hence $u_{1} \leftrightarrow u_{2}$. above question.

Alternatively, we say there exists a pathway if the following condition is met. There exists a concept $v$, such that $v$ in state $q \in \Sigma_{v}$ is a context for concept $u$ in state $p \in \Sigma_{u}$. We call it $e_{q} \in \mathcal{M}_{u}$. $e_{q}$ changes $p$ to a new state of concept $u$. This state is $q \wedge p \in \Sigma_{u}$, and $\mu\left(p \wedge q, e_{q}, p\right) \approx 1$.

\subsection{The Chaining and Integration of Pathways}

The above-mentioned ways of forging conceptual structure work by way of changing the set $\mathcal{M}$ of contexts associated with a given concept. For example, consider a SCOP that consists of the concepts STONE and ANIMAL and instances of ANIMAL, such as LION, TIGER, and MONKEY. We model an episode involving exposure to a lion made of stone as the concept combination STONE LION. The set of contexts associated with other animal concepts expands to include the potentiality that the animal can be made of stone. Thus $\mathcal{M}$ for MONKEY now includes STONE MONKEY and $\mathcal{M}$ for TIGER now includes STONE TIGER.

We said that with respect to a concept, the change of state $p \in \Sigma$ to state $q \in \Sigma$ under the influence of context $e$-hence for which $\mu(q, e, p)$ is the 
probability - can can be internally driven, externally driven, or both. This is also the case with respect to the context that drives a change of cognitive state; it may consist of a perceived stimulus or component(s) of the environment, or elements of the associative memory or worldview, or a mixture of the two. Pathways allow the autonomous (hence internally driven, not dependent upon change in the external environment) transformation of thoughts and ideas.

Instances of self-triggered recall can be chained together recursively resulting in a stream of thought. A change from one cognitive state $u \in \mathcal{U}$ to another $v \in \mathcal{U}$ may in turn modify the worldview, hence the (internally driven component of the) context by which $v$ is 'measured' or made sense of. Although $u$ may have been an eigenstate with respect to the context provided by the previous state of the worldview, $v$ may well not be an eigenstate with respect to the context provided by this newly updated version of the worldview. This means that there is another collapse or change of state from $v$ to $w$. This can continue recursively, until the concept enters a state that is an eigenstate with respect to the context provided by the worldview due to the effect on it of the previous cognitive state. It is through this chaining of episodes that indirect pathways or what we will refer to as routes through associative memory are forged.

$x, y, z \in \mathcal{X}$ can be said to be integrated if for each episode there exists some possible direct or indirect route to any other episode by way of pathways. If this is the case the whole exhibits emergent structure that cannot be reduced to the sum of the parts.

Thus conceptual structure arises by way of the creation of new concepts through abstraction and concept combination, and the creation of new pathways amongst episodes and concepts through processes such as associative recall, spatiotemporal contiguity, and the identification of natural relations. Examples of the formation of conceptual structure will be elaborated in the tentative model of the transition to cognitive modernity proposed in the pages to come.

\section{Modeling the Episodic Mind}

Now that the basic elements of SCOP and the extension of it to describe conceptual integration have been introduced, let us apply it to the subject of this paper: evolution of the capacity to develop an internal model of the world, or worldview, the components of which are accessed spontaneously, recursively, and in a context-sensitive manner to interpret (and generate) elements of culture. We begin by modeling the mind for which this is not the case.

\subsection{Coarseness of Memory Limits Associative Recall and Conceptual Structure}

The episodic mind may be innately predisposed to categorize some situationssuch as for example situations involving a member of another species - as instances of FRIEND, FOE, OR FOOD (without possessing words for these con- 
cepts). This facilitates the formation of pathways due to spatiotemporal contiguity. An experience that might not have evoked an episode encoded in memory if it had not been encoded as an instance of FRIEND might evoke it if it is encoded as an instance of FRIEND because the fact that both are instances of FRIEND makes the similarity more salient. Thus innate concepts facilitate the formation of pathways through associative recall, and thereby allow for a limited development of conceptual structure.

Although the episodic mind is capable of associative recall-for which the context that causes the change of cognitive state is something in the externally perceived world - it cannot engage in self-triggered recall - for which the context is generated internally. This means that instances of associative recall are not chained together recursively to give a stream of self-triggered thought. For this reason, the episodic minds of early hominids tended not to deviate far from the 'here and now' of perceived episodes, as opposed to the world of mental constructs and imagination. Why might our early ancestors have been incapable of self-triggered recall? It is reasonable to assume that the smaller brain of the early hominid had fewer neurons, and thus $n$ was small; that is, with few properties or subsymbolic microfeatures distinguishing one episode or item encoded in memory from another. Thus episodes were encoded in less detail. Since associative recall requires similarity of features or conceptual structure, and since there are fewer features, there are fewer routes for associative recall (in which one episode evokes another). On those rare occasions when associative recall does occur, it is unlikely to occur immediately afterward, and even if it does, since the context that causes it is external as opposed to internal, the result is not refinement or honing of the current thought. As mentioned earlier in the discussion of Figure 2, as we move through Hamming space away from the equator toward either $X$ or its antipode, the probability of encountering a memory item falls off sharply by a maximum (depending on the number and nature of items encoded) of $\sqrt{n} / n$. For the episodic mind, the region of substantial overlap between $X$ and another episode is extremely small.

The situation is compounded by the fact that since two processes by which new concepts come about, abstraction and conjunction, depend on associative recall, episodes are less apt to be conceptualized, or framed in terms of their conceptual structure. Since unconceptualized episodes lack conceptual structure they are not as deeply rooted into the worldview; thus there are few possible retrieval routes. As a simple example, an episode involving a ball but not conceptualized as an instance of TOY would not evoke memories of other toys such as dolls because the similarity between balls and dolls resided primarily at the conceptual level, not the perceptual level. Therefore there are few ways in which episodes can be similar not just at the property level but at more abstract conceptual levels. 


\subsection{Associativity is Detrimental for a Coarse-grained Mem- ory}

The occasional episodic mind might (due to a genetic mutation) have a higher tendency toward associative thought, i.e. a lower $\phi$, meaning that a recalled episode need not be very similar to the current episode to be evoked by it. Such an individual would be capable of making associations, perhaps even recursively, i.e. engaging in a kind of representational redescription, or stream of self-triggered thought. However, recall from section 3.1 Langton's (1992) finding that the information-carrying capacity of a system is maximized when its interconnectedness or self-similarity of components falls within a narrow regime between order and chaos. Forcing this kind of interaction between concepts in a coarse-grained memory (one with low $n$ ) would cause discontinuity between one thought and the next. Therefore thought would readily become chaotic, lacking the necessary continuity to hone a solution to a problem or develop and carry out a plan. Abstract thought, unlike episodic thought, cannot rely on the continuity of the external world (e.g. if a desk is in front of you now it is likely to still be in front of you now) to lend coherence to conscious experience;

this continuity must be internally driven. Since when $n$ is low, a mutation that results in a high $\phi$ leads to discontinuous (garbled) thought, it would be evolutionarily selected against.

\subsection{Coarse-grained Memory Lacks Quantum Structure}

In summary, since the episodic mind contains only coarse-grained episodes and few concepts, there is little opportunity for interaction and transformation of conceptual structure through reminding events, abstraction, or concept combination. So for any $u \in \mathcal{U}$, represented by the $\operatorname{SCOP}\left(\Sigma_{u}, \mathcal{M}_{u}, \mathcal{L}_{u}, \mu_{u}, \nu_{u}\right)$, any $p \in \Sigma$, for any context $e, f, g \ldots \in \mathcal{M}, \mu(p, e, p)$ is close to an eigenstate, hence $\approx 1$. One does not need the formalisms of quantum mechanics to model the dynamics of the episodic mind precisely because concepts are few, and have little effect on one another. (Note that SCOP can still be used to describe the episodic mind because as mentioned earlier it can describe both classical and quantum dynamics.)

\section{The Transition from Episodic to Mimetic Mind}

We noted that around 1.7 MYA there was a sudden increase in brain size, and this period is also associated with the origin of culture, and onset of what is referred to as the mimetic mind (Donald, 1991). The mimetic mind can access memories independent of external cues and act them out, group episodes together as instances of an abstract concept, refine ideas, and improve skills through repetition or rehearsal. We now proceed to examine a tentative scenario for how this transition came about. 


\subsection{Associativity is Beneficial for a Fine-grained Memory}

It seems reasonable to assume that the larger brain of the mimetic mind has more neurons than the brain of the episodic mind, and thus responds to more properties or subsymbolic microfeatures of episodes. Therefore episodes are encoded in more detail, i.e. $n$ is larger. There are more ways to distinguish one episode or item encoded in memory from another, and more pathways by which one episode can evoke representational redescription or a reminding of another episode. Thus it is well possible that for any given context $e, f, g \ldots \in \mathcal{M}$, any $p \in \Sigma$ is not an eigenstate, i.e., $\mu(p, e, p)<<1$.

Let us now examine what would happen if, again, due to a random mutation, a larger brained mimetic individual had a heightened tendency toward associative thought, i.e., a high $\phi$. As with the episodic mind, this means that a recalled episode need not be very similar to the current episode to be evoked by it, and the individual is capable of representational redescription, or selftriggered thought. One thought is able to spontaneously evoke another thought that is a modified version of the first, and so forth recursively, such that the original thought transforms through interaction with the individual's conceptual structure. However, since memory is not coarse but fine-grained (large $n$ ), the outcome is different. The Gaussian distribution of possible Hamming distances between the current episode and an item encoded in memory (as depicted in Figure 2) is wider, meaning there are now many more ways for an item in memory to be somewhat similar or loosely correlated to a given episode. This means that a stream of thought can retain the necessary balance between change and continuity to hone a solution to a problem or develop and carry out a plan. Since when $n$ is large a mutation that decreases $\phi$ thereby increasing $\mu$ leads to enhanced capacity for fruitful abstract thought, it facilitates a wide range of cognitive skills, and would be evolutionarily selected for.

Because the individual with a higher $\phi$ is more prone to associative recall, this individual is better equipped to form concepts. Since there is more conceptual structure in place, there is more of it to be affected by any given episode, and it is more likely that conceptual structure thereby contributes to the context that evokes a successive change of state (self-triggered recall).

\subsection{Onset of Extensive Abstraction}

The next step toward an integrated worldview is the formation of abstract concepts, such that items that share properties become conceived of as instances of the same thing. Suppose there are two episodes or concepts $u, v \in \mathcal{U}$, both modeled respectively by $\operatorname{SCOPs}\left(\Sigma_{u}, \mathcal{M}_{u}, \mathcal{L}_{u}, \mu_{u}, \nu_{u}\right)$ and $\left(\Sigma_{v}, \mathcal{M}_{v}, \mu_{v}, \nu_{v}\right)$. Suppose there exists a concept $w \in \mathcal{U}$, such that $u$ and $v$ are exemplars (instances) of $w$, and hence can be represented as states of $w$. Suppose that concept $w$ is modeled by a $\operatorname{SCOP}\left(\Sigma_{w}, \mathcal{M}_{w}, \mathcal{L}_{w}, \mu_{w}, \nu_{w}\right)$. Hence there are states $p, q \in \Sigma_{w}$, such that concepts $u$ and $v$ are states of $w$. We say that there exists a pathway from concept $u$ to concept $v$ and a pathway from concept $v$ to concept $u$, and 
denote these pathways $u \rightarrow v$ and $v \rightarrow u$. This can be generalized by saying that states $p_{u}$ of $u$ and $p_{v}$ of $v$ are also states of $w$.

Let us now examine how conceptual structure forms, starting with basic level concepts and moving toward concepts that are more abstract (Rosch, 1978) via the incorporation of new states and new contexts. Initially each concept has so few states associated with it that a state of one concept is rarely also a state of another concept. Thus reminding events are rare. However the activation of states of one concept that are also states of another is facilitated by the fact that the properties of any concept can themselves become viewed in conceptual terms, i.e. they can become concepts. Consider the concept MONKEY. A mimetic individual might encode in memory a state $p$ of MONKEY with the property $a$ : 'has arms', i.e. the state MONKEY WITH ARMS. If the individual has not just recognized that 'has arms' is a property of MONKEY but realized ARMS as a concept in its own right, then through the encoding of $p, \mathrm{MON}-$ KEY WITH ARMS, the concept MONKEY becomes connected to the concept ARMS. The conceptualization of an episode as MONKEY WITH ARMS provides a pathway from episodes related to monkeys to episodes related to arms. For the combination MONKEY ARM, the concept MONKEY functions as a context rather than as an entity. This is clear because if we consider the states that MONKEY ARM can be in, these states are not states of MONKEY, but they are states of ARM. (Clearly MONKEY ARM is not a MONKEY, but MONKEY ARM is an ARM.) To model this, we let $\mathcal{H}$ be the Hilbert space of states of ARM and $|u\rangle$ be the ground state of ARM. Then MONKEY is described by an orthogonal projection operator $P: \mathcal{H} \rightarrow \mathcal{H}$, which is a linear function, which is idempotent, i.e., $P^{2}=P$, and Hermitian, i.e., $P^{*}=P$, such that the state of MONKEY ARM is given by $P|u\rangle / \| P|u\rangle \|$ where $\| P|u\rangle \|$ is the length of $P|u\rangle$. (It is possible to imagine a context where it would be better to consider MONKEY as the entity. Of course, knowing the rules of the English language, the combination should then be written as ARM MONKEY, and it would mean a type of monkey characterized for example by a special ways of using its arms. It is a characteristic of metaphor that contexts are pushed into becoming entities.)

Our mimetic individual might subsequently encode a state of MONKEY with the property $b$ : 'has arms and legs'. The concept MONKEY is now connected to both concepts ARMS and LEGS. Moreover, 'has arms' and 'has arms and legs' are connected by the relation $b \leq a$. Thus an early step in the forging of organizational structure amongst the items encoded in memory is the grouping together of those properties that exhibit spatiotemporal contingency. When the properties 'has arms' and 'has legs' co-occur, they start to be conceived of as parts of a whole. This organizing of stimuli into sets of properties that tend to go together in the world is an important step toward the establishment of conceptual structure that internally models that world. As episodes accumulate in memory, so do states and their SCOP structures. As memory comes to encompass more of the natural relations amongst concepts, connections amongst concepts become more widespread, and an integrated conceptual structure starts to emerge. 
The same thing happens with contexts (as one might expect, since as stated earlier concepts can also be contexts). Consider the concept STAR. One might encode in memory a state of STAR under the context $e$ : to the north. The concept STAR is thereby connected to the concept NORTH. One might subsequently encode a state of STAR under the context $f$ : to the north at dusk. The concept STAR is now connected to the concept DUSK as well as the concept NORTH, and the contexts $e$ : to the north and $f$ : to the north at dusk are connected by the relation $f \leq e$. As memory comes to encompass contexts that are increasingly stronger, connections amongst concepts become more widespread, and the worldview thereby becomes more integrated.

As concepts accumulate, episodes are increasingly interpreted in terms of them, making episodes increasingly likely to evoke reminding of previously encoded episodes, or bring about representational redescription of the current episode. The probability increases that a given episode is similar enough to at least one item in memory to evoke it. With SCOP this is modeled as an increase in the number of potential contexts for which $\mu\left(p_{i}, e_{j}, p_{k}\right)>0$. The upshot is that one is able to recursively modify and redescribe thoughts and events. As more concepts become available, this gives rise to self-triggered sequences of thought, which increase in duration and frequency.

The probability of one MONKEY episode evoking another is enhanced through the recognition that they are in essence the same thing, i.e. instances of the concept MONKEY. The concept MONKEY facilitates the evoking of memories involving instances of monkeys. It connects any given monkey episode with not just other instances involving monkeys but with whatever associations have been formed amongst the concept MONKEY and other items (such as ARMS, LEGS...). Since representational redescription is now possible, using SCOP the function $\mu(q, e, p)$ gives a probability greater than zero that a particular state under a particular context will change to a particular other state, e.g. LARGE MONKEY might transform to DANGEROUS LARGE MONKEY.

A new state of a concept constitutes a new episodes, hence with each newlyformed state of a concept the number of episodes increases. If it is an episode that does not map onto a particular thing or event in the world but an abstraction (such as FURNITURE, or BEAUTY, or DEMOCRACY), it increases not just the number of items amongst which one could form associations, but the density of connections amongst previously encoded items. For example, due to a memory of someone using twine to strangle something, TWINE may be associatively linked to ARROW though they share few properties, because they are both instances of WEAPON. Depending on the context, a concept such as WEAPON can project onto states with different properties and different meanings. It is because there is greater potential in the mimetic mind for interaction amongst episodes (both conceptualized and unconceptualized) that a Hilbert space quantum model is well suited to model its dynamics. 


\subsection{Strengths and Limitations of the Mimetic Mind}

It is tentatively proposed that this state of affairs describes a mimetic mode of cognition. It is not merely a relay-station for coordinating inputs to appropriate responses. Because $n$ is higher, $\phi$ can afford to be lower, which facilitates the forging of meaningful conceptual structure. The mimetic mind engages extensively in abstraction and is capable of self-triggered recall. Since memories and concepts are starting to be organized in a way that reflects abstract relationships amongst them, reminding events are not infrequent, and potentially chained to give a short stream of self-triggered thought, and the current thought can become part of the context in which the next is experienced; thus actions can be refined. However, the mimetic mind is not very creative, and it is not integrated. it does not constitute a coherent mental model of how various aspects of the world relate to one another.

\section{The Transition from Mimetic Mind to Mod- ern Human Mind}

The archaeological record reveals a burst of creativity indicative of a cultural transition in Europe between 60,000 and 30,000 ka (Bar-Yosef, 1994; Klein, 1989; Leakey, 1984; Mellars, 1973, 1989a, b; Mithen, 1996, 1998; Soffer, 1994; Stringer \& Gamble, 1993; White, 1982). More recently it has been suggested that modern cognition originated earlier and more gradually in Africa, and that the European data reflects a wave of immigration of humans from Africa with sufficient intelligence and creative problem solving skills to cope with the new environment (McBrearty \& Brooks, 2000). Leakey (1984) writes "Known as the Upper Paleolithic Revolution, this collective archaeological signal is unmistakable evidence of the modern human mind at work" (p. 93-94). Mithen (1996) refers to this period as the 'big bang' of human culture, claiming that it marks the beginning of art, science, and religion, and that it shows more innovation than the previous six million years of human evoluti on. What brought about this cultural transition?

One possibility is that the cultural transition of the Middle/Upper Paleolithic reflects an enhanced ability to blend concepts (Fauconnier \& Turner, 2002). In a similar vein, Mithen (1998) suggests that it reflects onset of the capacity to transform conceptual spaces. The answer proposed here is consistent with these hypotheses but further specifies what kind of cognitive structure could have arisen to allow for these abilities.

\subsection{Onset of the Capacity to Shift between Analytic and Associative Thought}

It is suggested that the transition from a mimetic to a modern form of cognition is what gave rise to the cultural transition of the Middle/Upper Paleolithic, and 
that this came about through onset of the capacity to subject different situations to different kinds of cognitive processing. It is widely believed that there are two forms of thought, or that thought varies along a continuum between two extremes (Ashby \& Ell, 2002; Dennett, 1978; Gabora, 2002a, b; Gabora, under revision; Johnson-Laird, 1983; Neisser, 1963, Piaget, 1926; Rips, 2001; Sloman, 1996). At one end of the continuum is an analytic mode of thought conducive to deduction and to describing and analyzing relationships of cause and effect. At the other end of the continuum is an intuitive, overinclusive, or associative mode of thought conducive to finding subtle relationships; i.e. connections between items that are correlated, but not necessarily causally related. In this mode, items are represented in terms of not just their most typical properties but also in terms of less typical, perhaps context-dependent ones as well. Associative thought is related to the notion of flat associative hierarchies, a term applied to those who give not only typical but also marginal or atypical items when asked to say words that come to mind in response to a particular word, e.g. OSTRICH in response to BIRD, or BEANBAG CHAIR in response to CHAIR (Mednick, 1962). The notion of two forms of thought is also captured in Gärdenfors' tiered model of cognition; the analytic mode is related to the symbolic level of cognition and the associative mode is related to the conceptual level.

It has been proposed that the transition from mimetic mind to modern human mind came about through the onset of contextual focus: the capacity to spontaneously focus or defocus attention in response to the current situation, and thus alternate between analytic and associative thought according to the demands of the situation (Gabora, 2003, Aerts \& Gabora, 2005a). This allowed for a worldview with recursively embedded hierarchical structure and concepts of varying levels of abstraction, which paved the way for conceptual integration. Let us now formulate this hypothesis more precisely.

Here we describe the onset of the capacity for contextual focus mathematically as the onset of the capacity to modulate $\phi$. It is proposed that analytic thought is the result of focusing attention, thereby increasing $\phi$, which globally (for all states of all concepts, as well as all unconceptualized cognitive states) decreases $\mu$, the transition probability. Since one state is less likely to spontaneously give way to another as a consequence of their shared properties and abstract conceptual structure, effort can be focused on manipulating items in their most prototypical, unambiguous form according to the rules of logic, as in the formation of abstract concepts, the recognition of episodes as instances of a concept, and furthering development of the structure of the lattices of properties and contexts associated with known, existing concepts as described above.

It is proposed that associative thought is the result of defocusing attention, thereby decreasing $\phi$. One is more likely to make associations and be reminded of items based on not just their most typical properties but also in terms of less typical, context-dependent properties, or on the basis of abstract structure, or spatiotemporal contiguity. This is conducive to applying known concepts in new contexts; for example, application of the concept HANDLE to challenging situations, as in 'get a handle on the situation' or to intimate relationships, as in 'love handle'. This results in a transition from domain-specific representa- 
tions to more broadly applicable representations, enabling seemingly disparate domains of knowledge to be bridged.

\subsection{The Formation of Conjunctions}

The capacity to temporarily decrease $\phi$ and thereby enter a more chaotic regime (for example, when one is stuck in a problem or seeking self-expression) is not just conducive to applying concepts in new contexts, but also to merging concepts to generate new ones. This is described as follows.

For two concepts $u, v \in \mathcal{U}$ described by $\operatorname{SCOPs}\left(\Sigma_{u}, \mathcal{M}_{u}, \mathcal{L}_{u}, \mu_{u}, \nu_{u}\right)$ and $\left(\Sigma_{v}, \mathcal{M}_{v}, \mu_{v}, \nu_{v}\right)$ it may be that a conjunction exists. A conjunction is described in SCOP using the tensor product when the SCOPs are Hilbert space based, as described in section 2.5. The conjunction is considered a new concept, not just the logical construct of a conjunction. For example, suppose $u$ is the concept SNOW and $v$ the concept MAN. The tensor product SCOP models the concept SNOWMAN. States of SNOWMAN are states of SNOW and states of MAN. For example OG WITH SNOW ON HIS HEAD, as one concept, is not a state of the concept SNOWMAN, but it is a state of the logical construct of the conjunction SNOW and MAN.

The formation of a conjunction involves two steps (Aerts \& Gabora, 2005b). The first step involves recognition that one ofthe concepts is a specific kind of context for the other. For example, consider the concepts SNOW and MAN. Note that The man is made of snow can be a context for MAN. It changes the ground state of MAN to a state of MAN that is a 'prelude' to the formation of the new concept SNOWMAN. This prelude state is the root of the pathway that connects the concepts SNOW and MAN.

We define this pathway formally as follows. Consider two concepts $u, v \in \mathcal{U}$, modeled by SCOPs $\left(\Sigma_{u}, \mathcal{M}_{u}, \mathcal{L}_{u}, \mu_{u}, \nu_{u}\right)$ and $\left(\Sigma_{v}, \mathcal{M}_{v}, \mu_{v}, \nu_{v}\right)$. Supposes that state $p \in \Sigma_{u}$ (hence a state of concept $u$ ) is a context of concept $v$, which we call $e_{p} \in \mathcal{M}_{v}$. This context $e_{p}$ creates a new state of concept $v$ by acting on the ground state $\hat{q}$ of $v$. We could for example denote this new state as $\hat{p} \triangle q$. We say that a pathway exists from $\hat{p}$ to $q$, denoted $\hat{p} \leftrightarrow q$. State $\hat{p} \triangle q$ is a prelude to the state of the new concept when a conjunction of $u$ and $v$ forms. For example, the state of MAN that results when the context the man is made of snow influences the ground state of MAN is the state $\hat{p} \triangle q$. It is what we are calling a 'prelude state' of the ground state of the new concept SNOWMAN.

\subsection{Conceptual Integration}

The formation of a new concept increases the number of concepts by one, but it increases the number of pathways by, not just the number of states of that concept, but also other episodes and concepts that share conceptual structure (e.g. BEANBAG CHAIR is a state of BEANBAG and a state of CHAIR and for that matter a state of FURNITURE...). Therefore, as the number of concepts increases, the number of pathways increases faster. It is known in graph theory 
that when the ratio of edges to points reaches approximately 0.5 - sometimes referred to as the percolation threshold (Kauffman, 1993) - the probability that one giant cluster emerges goes from extremely unlikely to almost inevitable (Erdos \& Renyi, 1959, 1960). Here, when the ratio of pathways to episodes and concepts surpasses this threshold, it becomes almost inevitable that the items in memory reach a critical density where a large subset of them undergoes a phase transition to a state wherein for each episode or concept there exists some possible direct or indirect route to any other episode or concept by way of internally driven remindings or associations. In this way, they constitute an integrated conceptual web.

There are numerous ways of achieving conceptual integration even starting from the same set of episodes, and these ways convey varying degrees of integration and consistency on the resulting worldview. They differ with respect to the lattice structures of states and contexts that connect these episodes, and the degree of penetration of the conceptual space. Under what conditions will the transformation from discrete memories to interconnected conceptual web actually occur? We need to show that some subset of the concepts encoded in an individual's mind inevitably reach a critical point where there exists a direct or indirect route (sequentially applied pathways) by which every member of that subset can access every other member. We need to show that $R$, the number of pathways by which one episode can evoke another, increases faster than $s$, the number of episodes. That is, as the memory assimilates episodes, it comes to have more ways of generating them than the number of them that has explicitly been encoded.

Under what conditions does $R$ increase faster than $s$ ? The ability to form context-sensitive concepts plays a crucial role. To determine how this affects $R$, let us assume for the moment that memory is fully connected. In other words, we temporarily assume that all states of all encoded items have a non-zero transition probability to all states of all other encoded items. Clearly this is not the case, but this simplification facilitates analysis that also applies to a more realistic model of memory. To make things simple we will be conservative and limit the sort of pathways under consideration to associative recall and pathways between abstract concepts and their instances. Concepts have $n$ properties, where the number of applicable properties (properties with non-zero weights) ranges from a minimum of $m$ to a maximum of $M . R_{A}$, the number of ways a pathway can be realized through abstraction, equals the number of pathways enabled by an $n$-dimensional concept, multiplied by the number of n-dimensional concepts, summed over all values of $n$ from $m$ to $M-1$. The number of pathways equals the number of items that are instances of an $n$-dimensional concept $=2^{M-n}$. The number of $n$-dimensional concepts is equal to the binomial coefficient of $M$ and $n$. The result is multiplied by two since an a concept can evoke an instance, and likewise, an instance can evoke a concept.

$$
R_{A}=2\left(2^{M-m}\left(\begin{array}{l}
M \\
m
\end{array}\right)+2^{M-(m+1)}\left(\begin{array}{c}
M \\
m+1
\end{array}\right)+\ldots+2\left(\begin{array}{c}
M \\
M-1
\end{array}\right)\right)
$$




$$
=2 \sum_{n=m}^{M-1} 2^{M-n}(n-1)\left(\begin{array}{c}
M \\
n
\end{array}\right)
$$

This shows that the greater the density of concepts, the greater the likelihood of conceptual integration. Abstraction (the formation of a general concept out of instances) increases $s$ by creating a new concept. But it increases $R$ more, because the more abstract the concept, the greater the number of concepts a short Hamming distance away (since $\left|x_{i}-k_{i}\right|=0$ for the irrelevant properties, so they make no contribution to Hamming distance). In other words, concepts with fewer properties enable exponentially more pathways.

A second thing to note is that as $n$ starts to decrease, the number of possible abstractions for each value of $n$ increases (up to $M / 2$ after which it starts to decrease). Thus the more likely any given memory item is to get activated and participate in a reminding or associative recall event. Whereas $R$ increases as abstraction makes relationships amongst concepts increasingly explicit, $s$ levels off as new experiences have to be increasingly unusual in order to result in the formation of new concepts. Furthermore, when the carrying capacity of the memory is reached (such that the encoding of new items entails significant degradation of previously encoded ones), $s$ plateaus but $R$ does not. Taken together these points mean: the more deeply a mind delves into abstractions, the more the distribution in Figure 2 rises and becomes skewed to the left. The effect is magnified by the fact that the more active a region of conceptual space, the more likely an abstraction is to be positioned there, such that abstractions beget abstractions through positive feedback loops.

Thus, as long as $\phi$ is low enough to permit abstraction and small enough to permit temporal continuity, the average value of $n$ decreases, and sooner or later, the system is expected to reach a critical percolation threshold such that $R$ increases exponentially faster than $s$. The memory becomes so densely packed that any episode is bound to be close enough in Hamming distance to some previously-encoded episode to evoke it. The memory (or some portion of it) is holograph-like in the sense that there is a pathway of associations from any one episode to any other. What was once just a collection of isolated memories is now a structured network of concepts, instances, and relationships - a worldview. It can engage in streams of thought that redefine elements of the world in terms of their substitutable and complementary relationships, plan a course of action, engage in symbolic communication with others, and so forth, as outlined in the introduction. It is used not just passively to understand what the world is like, but actively to navigate life experiences.

\subsection{Conceptual Integration in SCOP}

Let us now show what conceptual integration means with respect to SCOP. For an arbitrary subset $X \subset \mathcal{X}$ of episodes we define

$$
c l(X)=\{y \mid y \in \mathcal{X} \text {, and } \exists x \in \mathcal{X} \text { such that } y \leftrightarrow x\}
$$


In Appendix 1 we prove that $c l$ is a closure relation. This allows us to consider for an arbitrary subset of episodes $X \subset \mathcal{X}$ its closure $\operatorname{cl}(X)$. This is the set of episodes such that for each episode $x \in \operatorname{cl}(X)$ there is an episode $y \in X$ such that $x \leftrightarrow y$ is connected to $y$.

We now consider 'one' specific episode $x$, and the subset containing 'only' this one episode, hence the singleton $\{x\}$. We can ask what is the closure of this subset, hence what is $c l(\{x\})$ ? In other words, what is the set of episodes connected to $x$ ? In Appendix 2 we show that the closure relation conserves full connectedness. This means that if we start by considering a fully connected subset of episodes, and add all the episodes connected to episodes of this subset, this is still a fully connected subset of episodes.

The next thing we want to show is that $\operatorname{cl}(X)$ is the biggest fully connected subset containing $X$. This proof is given in Appendix 3. Let us investigate what this tells us about the set of all episodes $\mathcal{X}$. Consider two episodes $x, y \in \mathcal{X}$. There are theoretically only two possibilities. Either $x \leftrightarrow y$, or $x \leftrightarrow y$. If $x \leftrightarrow y$, we can consider $\operatorname{cl}(\{x, y\})$, which is then the biggest fully connected subset containing $x$ and $y$. If $x \leftrightarrow y$ we consider $\operatorname{cl}(\{x\})$ and $\operatorname{cl}(\{y\})$. We know that $\operatorname{cl}(\{x\})$ is the biggest fully connected subset containing $x$ and $\operatorname{cl}(\{y\})$ is the biggest fully connected subset containing $y$. Can $\operatorname{cl}(\{x\})$ and $\operatorname{cl}(\{y\})$ have elements in common? No, indeed, suppose that $z \in \operatorname{cl}(\{x\}) \cap \operatorname{cl}(\{y\})$. Then $z \leftrightarrow x$ and $z \leftrightarrow y$, and from transitivity of the connectivity relation it follows that $x \leftrightarrow y$. Hence $\operatorname{cl}(\{x\}) \cap \operatorname{cl}(\{y\})=\emptyset$. But the situation is more extreme. We do not even have any element in $\operatorname{cl}(\{x\})$ that is connected with any element in $\operatorname{cl}(\{y\})$. We can prove the proposition that for the closure of two single episodes, the closure subsets are equal, or their intersection is empty. This proof is given in Appendix 4. In Appendix 5 we prove that $\mathcal{X}$ is equal to the disjoint union of fully connected subsets of episodes, and moreover each of these fully connected subsets of episodes is the closure of a single episode. This implies that if one of these regions of fully connected subsets becomes dominant, in the sense that it is the one used to generate internal contexts and interpret episodes, and the one generally activated in conscious experience, then also this one is the closure of one single episode. This one single episode is not unique; there are many episodes that 'as one single episode' can play the role of being the generating element for the whole closure. This is the meaning of the possibility that $\operatorname{cl}(\{y\})=\operatorname{cl}(\{z\})$ is many occasions, and in fact whenever $y \leftrightarrow z$. Others may continue to exist but be virtually inaccessible from the dominant one, and may be experienced only in situations where there are fewer social and pragmatic constraints on how we internally generate the contexts that enable thoughts to unfold. The bottom line is that the conceptual structure now has a vast amount of potentiality, and the extent to which this potentiality is accessed at any given instant depends on the situation and the value of $\phi$. 


\section{The Development and Evolution of an Inte- grated Worldview}

It is possible that some items in memory are isolated outliers - not woven into this conceptual web, and thus inaccessible. (It seems reasonable that this might be the case for experiences that are far from everyday reality and insufficiently reinforced through reminding events, such as dreams or perhaps traumatic experiences.) Since we do not have conscious access to them, it is difficult to know whether they are abundant or rare. It is possible that much of what constitutes a mind is never woven into an integrated worldview. What we can say is that to constitute a worldview, the memory architecture must be such that, for any typically encountered stimulus or situation, there must exist a means of relating or re-describing it in terms of previously encoded items. Thus for an integrated worldview, this includes all memory items necessary for the redescription and accommodation of frequently encountered experiences.

Note that conceptual integration would be unlikely to be achieved simultaneously throughout a society of individuals. It would thus take time for social propagation of the cultural 'fruits' of such an achievement. However, even if for example one individual, Agu has a higher activation threshold than another individual, say Oga, once he has assimilated enough of Oga's abstractions, his concepts become so densely packed that a version of Oga's worldview snaps into place in his mind. Agu in turn shares fragments of his worldview with his friends, who in turn shares them with others. These different 'hosts' expose their versions of these fragments of what was originally Oga's worldview to different experiences, different bodily constraints, sculpting them into unique internal models of the world. Small differences are amplified through positive feedback, transforming the space of viable worldview niches. Individuals whose activation threshold is too small to achieve worldview closure are at a reproductive disadvantage and, over time, eliminated from the population. When Agu imitates a mannerism of Ogu's he might, on the fly, modify it by putting his own spin on it. As worldviews become increasingly complex, the artifacts they manifest in the world become increasingly complex, which necessitates even more complex worldviews, and so forth. Thus a positive feedback cycle sets in. The elements of human culture - the artifacts, the stories, the languages in which they are told - reflect the states of the worldviews that generated them, specifically the associative structure amongst concepts (and the words by which they are accessed), which in turn reflects their underlying motives and desires.

This analysis suggests that animals are not prohibited from evolving complex cognition a priori, but that without the physical capacity to generate and manipulate complex artifacts and vocalizations there is insufficient evolutionary pressure to tinker with $\phi$, the association threshold, until it achieves the requisite delicate balance to sustain a stream of thought, or to establish and refine the necessary feedback mechanisms to dynamically tune it to match to the degree of associativity or 'conceptual fluidity' required at any given instant. It may be that humans are the only species for which the benefits of this tinkering process 
have outweighed the risks. This would make sense because with the capacity for complex speech and hand movement there are clearly more ways to manifest complex thoughts, i.e., more ways in which they could yield outcomes in the world.

It was noted that it is not the presence of but the capacity for an integrated worldview that the human species has come to possess. Following the pioneering efforts of Piaget, Vygotsky and more recently others, it has become clear that a worldview is not present from birth but develops naturally through experience in the world. The infant mind is predisposed to selectively attend to biologically salient features, and respond accordingly. If it is hungry and sees its mother's breast, it suckles; if it feels something extremely hot or cold it pulls away, and so forth. In addition to innate predispositions to respond categorically to certain stimuli, it is widely thought that infants possess higher cognitive competencies (Gelman, 1993; Keil, 1995). These competencies may be due to core knowledge (Spelke, 2000), intuitive theories (Carey, 1985), or simply predispositions to direct attention to salient (particularly social) elements of a situation (Leslie, 2000). An infant is also capable of storing episodes as memories. Although episodes from infancy are rarely accessible later in life, they $d o$ get etched into memory, as evidenced by the capacity for reminding events, which is present by two month of age (Davis \& Rovee-Collier, 1983; Rovee-Collier et al., 1999; Matzel et al., 1992) and possibly earlier (Rovee-Collier, pers. comm). Thus, an infant is born predisposed toward conceptual integration, but the process must begin anew in each young mind. The infant may be born with innate concepts, but its memory is not densely packed enough (i.e., distributed representations do not overlap enough) to prompt recursive redescription or reminding events. Even if items are encoded in detail, that is, even if $n$, the mean number of properties per encoded item, is large, there are too few of them for reminding events to occur with high frequency. Returning to Figure 2, there are few items within a given Hamming distance of any item. Moreover, it lacks the conceptual structure that facilitates efforts to understand and make sense of how episodes and their contents are related to one another.

Although the issue is controversial, it is widely accepted that between six and eight years of age, a child moves from implicit, domain-specific representations to explicit, more broadly applicable representations (Karmiloff-Smith, 1990, 1992). Aided by social exchange, and mediated by artifacts, a framework for how things are and how things work falls into place in a child's mind, and it bears some likeness (and also some dis-similarities) to that of its predecessors, such as the worldviews of parents and other influential individuals. Some experiences are either so consistent, or so inconsistent with the child's worldview that they have little impact on it. Others mesh readily with existing ideas, or ring true intuitively, and percolate deep into the worldview, renewing the child's understanding of a myriad other notions or events. The child is thereby encultured, and becomes a unique $\operatorname{cog}$ in the culture-evolving machinery. Human life offers a continuous stream of situations that disrupt the state of conceptual integration. For example, problems arise that cannot be consistently explained in terms of other elements of the worldview as they are currently understood. The 
individual may reconceptualize the problem or situation from the perspective of a context generated by the worldview. The process is recursive in that the new cognitive state is a function of both the previous cognitive state and the context. Each internally generated context may induce a change in the conception of the problem or situation, and each change in conception of the problem may modify he worldview, until it eventually recoups the state of conceptual integration.

The idea of a conceptually integrated worldview sheds light on the thorny issue of the role of evolution in cognition. Hampton (2004) argues that if evolutionary psychology as a form of strong adaptationism insists that novel behaviors are the product of directly selected adaptations, it follows that there can be no evolutionary account of many modern activities such as driving and reading. However, one of the four approaches subsumed under by evolutionary psychology, as laid out in The Adapted Mind (Barkow et al. 1993), is the study of culture as an evolutionary process in its own right. Even if modern activities defy explanation in terms of biological evolution they may be explicable in terms of cultural evolution. As Deacon (1997) points out, language evolution is probably thousands of times more rapid than brain evolution. Bouissac (1998) concludes from this that "such a parasitic theory of language evolution is rooted in Dawkins' meme hypothesis". However, the notion of conceptual integration suggests an alternative: that it is not discrete memes (transmittable ideas or artifacts) that evolve through culture, but conceptually integrated networks of them-worldviews. The argument for this is laid out in detail elsewhere (Gabora 2004); here it is summarized insofar as is necessary and relevant.

A basic tenet of Dawkins' proposal is that the meme is replicator-an entity that makes copies of itself. Clearly ideas and artifacts are not replicators of the same sort as modern day organisms, which use a DNA- or RNA- based self-assembly code. A telltale sign of self-assembly coded replication is restricted inheritance of acquired characteristics. The effects of contextual interactions between the organism and its environment are wiped out in the next generation, and do not affect the evolution of lineages. Thus for example, if one cuts off the tail of a mouse, its offspring have tails of normal length. As many have noted, however, acquired characteristics may be inherited in cultural transmission. If you hear a joke and then put your own slant on it in the retelling of it, those who pass it on subsequently will likely pass on your changed version.) An idea may retain structure as it passes from one individual to another (like a radio signal intercepted by a new radio), but does not replicate it.

Cultural artifacts are not the only structures to have evolved without a selfassembly code. Our application of closure to the origin and evolution of an integrated worldview was inspired by current thinking on the problem of how life began (Gabora, 2006; Kaufman, 1993; Morowitz, 1992; Vetsigian, Woese, \& Goldenfeld, 2006; W?echtersh? euser, 1992; Weber, 1998, 2000; Weber \& Depew, 1996; Williams \& Frausto da Silva, 1999, 2002, 2003; Woese, 2002). For example, Kaufman (1993) uses the concept of autocatalysis to describe how the earliest self-replicating structures could have emerged. The autocatalytic sets of polymers widely believed to be the earliest form of life generated self-similar structure, but since there was no genetic code yet to copy from, there was no ex- 
plicit copying going on. The presence of a given catalytic polymer, say polymer $\mathrm{X}$, simply sped up the rate at which certain reactions took place, while another catalytic polymer, say Y, influenced the reaction that generated X. Eventually, for each polymer in the set, there existed a reaction that catalyzed it. Thus the set as a whole was autocatalytic, able to replicate itself.

${ }^{5}$ These earliest life forms have been shown to have evolved through a nonDarwinian process involving not natural selection operating at the population level but communal exchange of innovations operating at the individual level.

Kauffman (1993) argues that it is extremely unlikely that the earliest life forms themselves replicated using a self-assembly code, though such a code could have arisen by way of a simpler, uncoded self-replicating structure. His proposal is that the first living organism was a set of autocatalytic polymers. Since there were no self-assembly instructions to copy from, there was no explicit copying going on. The presence of a given catalytic polymer, say X, simply speeded up the rate at which certain reactions took place, while another polymer, say $\mathrm{Y}$, influenced the reaction that generated X. Eventually, for each polymer, there existed another that catalyzed its formation. Such autocatalytic sets have been referred to as primitive replicators because they generate self-similar structure, but in a self-organized manner, through bottom-up interactions rather than a top-down self-assembly code (such as the genetic code), thus they replicateor more precisely, regenerate themselves-with low fidelity (Gabora 2004, 2006, 2008). They are self-mending in the sense that if the web of catalytic reactions breaks down (if, for example, one of the polymers gets used up) one or more others is eventually found to take its place, such that the network of reactions becomes again able to regenerate itself.

It may be that the emergence of this kind of complex, adaptive self-modifying, self-mending, self-regenerating (i.e., autopoietic) structure is the critical step in the origin of any kind of evolutionary process, be it biological, cultural, or some other sort that we may not have identified. The evolution of culture involves not just creativity, nor just imitation, but a proclivity to put our own spin on the inventions of others, to use them for our own purposes and adapt them to our own needs and desires, such that the process becomes cumulative, adaptive, and open-ended. The present paper describes steps toward the kind of cognitive structure that is capable of supporting this process. Like Kauffman's (1993) autocatalytic sets of polymers, an integrated worldview is autopoietic. Just as polymers catalyze reactions that generate other polymers, the retrieval of an item from memory can in turn trigger other items, cross-linking mem-

\footnotetext{
${ }^{5}$ Some authors (e.g. Thompson, 2007) claim that life requires a semi-permeable boundary to allow for spatial demarcation and containment. We believe this is unnecessary because in an autocatalytic network, if there is a molecule that catalyzes a particular molecule X, then molecule X can effectively enter and become part of the network. If there is no molecule that catalyzes molecule $\mathrm{X}$, then molecule $\mathrm{X}$ is effectively excluded. Possession of a membrane boundary thus strikes us as a redundant, arbitrary requirement, true of life as we know it but not life as it could be; one can imagine entities that are alive but nonetheless interwoven without clear-cut physical boundaries separating them. Nevertheless it is indeed the case that semipermeable membranes play an exceedingly important role in life and may turn out to be a requirement.
} 
ories, ideas, and concepts into an integrated conceptual structure. Moreover, this conceptual structure regenerates itself; it constitutes a primitive replicator. Its elements (ideas, stories, and so forth) evoke one another through deduction, association, and reminding events, frequently resulting in their expression in the form of cultural artifacts, or get communicated and incorporated into the worldviews of other individuals. The result is that contextual interactions can affect the 'cultural lineage' downstream and thus play an important role in their evolution. Thus a worldview gets regenerated in a piecemeal fashion through social learning, both directly and mediated by artifacts. Ideas and artifacts are not full-fledged replicators themselves, but manifestations of a worldview, which is a primitive replicator. They participate in the evolution of culture by revealing certain aspects of the worldview that generated them, thereby affecting the worldviews of those exposed to them.

In Kauffman's (1993) computer simulations of the origin of life through autocatalytic closure, each polymer was composed of up to a maximum of $\mathrm{M}$ monomers, and assigned a low a priori random probability $P$ of catalyzing each reaction. The lower the value of $P$, the greater $M$ had to be, and vice versa in order for autocatalytic closure to occur. Computer simulation of conceptual integration might be expected to reveal a similar trade-off between $M$, which in the cognitive scenario refers to the maximum number of dimensions along which episodes are encoded, and $\phi$, the probability for items in memory to evoke one another. We might also expect to see that, as with biological life, worldviews that replicate themselves more efficiently will proliferate at the expense of others that are less efficient. Indeed, we are compelled to engage in behavior that helps others absorb the knowledge, attitudes, and ways of doing things that constitute our worldview, and those that were not so inclined in the past may well have been selected against at this cultural level.

\section{Summary and Discussion}

Our capacity to adapt ideas to new situations, see one thing in terms of another, blend concepts together in an endless variety of ways to interpret and express real or imagined situations, are all indicative of the integrated nature of a human worldview. This paper has presented a speculative attempt to formalize the process by which conceptual integration comes about. The paper does not tell a finished story but merely lays some groundwork and points the way. Several elements will require further development, such as the use of the Schrödinger equation to model the dynamical unfolding of the conception of combinations of concepts.

Let us retrace the major steps taken. The integrated quality of a worldviewthe capacity for interpenetration of one thought or idea into another-presents a chicken and egg paradox. Until memories are woven into an integrated worldview, how can they generate the reminding or evoking events that constitute a stream of thought? Conversely, until a mind can generate streams of thought, how does it integrate memories into a worldview? How could something com- 
posed of complex, mutually dependent parts come to be? Moreover given the chameleon-like behavior of concepts, and what kind of mathematical description can we give to them? In this paper it is proposed that a worldview emerges through conceptual integration yielding a structure whose dynamics requires the quantum formalism for its description.

Let us retrace the steps in how closure and SCOP are used to model the transition to an integrated worldview. The associative structure of the episodic minds of Homo habilis, our earliest ancestors was coarse-grained; items were not encoded in detail. Thus we model the Homo habilis mind as having little interaction amongst concepts, little opportunity for reminding events and associations, no pressing need for a quantum formalism, and no conceptually integrated worldview.

The memory of the mimetic mind of Homo erectus is more fine-grained; items are encoded in greater detail. Thus there is opportunity for reminding events and associations, and interaction amongst concepts, and a need for the quantum formalism to model how concepts emerge from a ground state to an actual state in a manner that depends on the constellation of other evoked concepts. There is concept formation through abstraction, modeled as the recognition that episodes or concepts are instances of a more general concept, and the accumulation of sets of states, contexts, properties, weights and transition probabilities. There is formation of conceptual structure modeled as the formation of lattices of properties and contexts through the realization of natural relations. However, because there is no ability to modulate the degree of interaction amongst concepts depending on what is called for by the situation, the conceptual web is crude and fragmented.

The modern human mind has the ability to shift between analytic thought, conducive primarily to realizing relationships amongst states of a known concept, and associative thought, conducive primarily to forging new concepts through the formation of conjunctions, which are entangled states that result through application of the tensor product of the Hilbert spaces of the two constituent concepts. It is proposed that the penultimate step toward achieving an integrated worldview was to acquire the capacity to spontaneously focus attention (conducive to analytic thought) or defocus attention (conducive to associative thought) depending on the circumstance. This is modeled as onset of the modulation of $\mu$, the transition probabilities using a variable we called $\phi$. Once the capacity has evolved to alter $\phi$ according to the situation, analytic thought and associative thought can work in concert to organize and reorganize conceptual structure. Analytic thought enables the identification of causal relationships, while associative thought facilitates recognition of items in memory that are correlated, i.e. that share properties, which in turn provides more ingredients for analytic thought. Many species can learn, imitate, remember, and perhaps even form concepts. So this capacity to focus or defocus may be the key step in the attainment of complex language, religion, science, art, and other aspects of culture that make us unique. Defocused thought forges connections amongst items that share a deep structure but are superficially unrelated. Since these relations are often difficult to find, cultural learning also plays a key role 
here, filling in the missing links that the child does not find on its own (perhaps the vast majority). Because most of the fruits of such creative achievements are culturally transmitted, it is not necessary that we each individually generate an interconnected worldview from scratch. A hard-to-come-by concept or idea need only be realized in the mind of one individual; the other members of a society get it 'for free' without any particularly focused or defocused thought.

As episodes accumulate in memory, and concepts acquire more states, the set of concepts grows and becomes more connected. When the same set of concepts arise repeatedly, concepts themselves form more abstract concepts, giving rise to semantic hierarchies. The probability increases that any experience triggers an episode encoded in memory which in turn triggers another, and so forth, generating a stream of thought. Streams of thought facilitate the formation of yet more abstract concepts. When the ratio of cognitive states to concepts reaches approximately $2: 1$, the probability becomes almost inevitable that concepts 'join' in the sense that there come to exist cognitive states that are states of more than one concept. At this point, for any episode in the dominant subset of episodes there exists a possible route, or sequence of associative pathways (however indirect) to any other there exists a possible associative path (however indirect) to any other, enabling new experiences to be framed in terms of previous ones. We became able to encode, interpret, and reinterpret episodes using constellations of concepts of varying degrees of abstraction, wherein the meaning of each concept in the constellation shifts depending on the context provided by other simultaneously evoked concepts. Together they form a conceptual web that from the inside is experienced as an integrated model of the world, or worldview. It has been suggested that it is the conceptually integrated worldview that constitutes the replicating unit of cultural evolution. Like a living organism, a worldview may naturally be drawn to gaps or inconsistencies and attempt to heal them (Gabora 1999, 2008). Elaboration of this approach may provide formal models of such psychological phenomena as repression, fragmentation, and integration.

\section{Acknowledgments}

This research is supported by grants from the Social Sciences and Humanities Research Council of Canada and the Research Council of the Free University of Brussels, Belgium. Part of the paper was written during a year spent by the first author at the PACE Center, Tufts University.

\section{Appendix 1}

A closure relation is extensive, monotone and idempotent. This means that for $X, Y \subset \mathcal{X}$ we have

$$
X \subset \operatorname{cl}(X) \text { extensive }
$$




$$
\begin{aligned}
& X \subset Y \Rightarrow \operatorname{cl}(X) \subset \operatorname{cl}(Y) \quad \text { monotone } \\
& \operatorname{cl}(\operatorname{cl}(X))=\operatorname{cl}(X) \quad \text { idempotent }
\end{aligned}
$$

Proof: Consider an episode $x \in X \subset \mathcal{X}$. Since $x \leftrightarrow x$ this episode is connected to an episode $x \in \mathcal{X}$, hence, $x \in \operatorname{cl}(X)$. This proves (17).

Let us prove (18). Suppose there exist two subsets of episodes $X, Y \subset \mathcal{X}$ such that $X \subset Y$, and an episode $x \in \operatorname{cl}(X)$. Since $x \in \operatorname{cl}(X)$ this means that there exists an episode $y \in X$ such that $x \leftrightarrow y$. From $X \subset Y$ it follows that $y \in Y$. Hence, there exists an episode $y \in Y$ such that $x \leftrightarrow y$, which proves that $x \in \operatorname{cl}(Y)$. Hence, this shows that $\operatorname{cl}(X) \subset \operatorname{cl}(Y)$. This proves (18).

Let us prove (19). From (17) we know that $X \subset \operatorname{cl}(X)$, and applying (18) we get $\operatorname{cl}(X) \subset \operatorname{cl}(\operatorname{cl}(X))$. This means that to prove (19) we only need to show that $\operatorname{cl}(\operatorname{cl}(X)) \subset \operatorname{cl}(X)$. Consider $x \in \operatorname{cl}(\operatorname{cl}(X))$. This means that there exists an episode $y \in \operatorname{cl}(X)$ such that $x \leftrightarrow y$. Since $y \in \operatorname{cl}(X)$, there exists an episode $z \in X$ such that $y \leftrightarrow z$. Applying the transitivity of the 'connection relation' we get that $x \leftrightarrow z$, which means that $x \in \operatorname{cl}(X)$. Hence, we have shown that $c l(c l(X)) \subset \operatorname{cl}(X)$, and we have proven (19).

\section{Appendix 2}

Let us prove that the closure relation conserves full connectedness. We start by stating that a subset of episodes $X \subset \mathcal{X}$ is fully connected if

$$
\forall x, y \in X \text { we have } x \leftrightarrow y
$$

Proposition: $\operatorname{cl}(\{x\})$ is a fully connected set of episodes.

Proof: Consider $y, z \in \operatorname{cl}(\{x\})$, then we have $y \leftrightarrow x$ and $z \leftrightarrow x$, and hence from the transitivity property of the 'connection relation' follows that $y \leftrightarrow z$.

Proposition: If $X$ is a fully connected set of episodes, then also $\operatorname{cl}(X)$ is a fully connected set of episodes.

Proof: Consider $x, y \in \operatorname{cl}(X)$, then there exists $z, t \in X$ such that $x \leftrightarrow z$ and $y \leftrightarrow t$. Since $X$ is a fully connected set, we have $z \leftrightarrow t$. Hence, from the transitivity of the 'connection relation' it follows that $x \leftrightarrow t$, and therefore $x \leftrightarrow y$. This proves that $\operatorname{cl}(X)$ is fully connected.

Hence, we have proven that the closure relation conserves full connectedness. 


\section{Appendix 3}

Proposition: If $X$ is a fully connected set of episodes, then $\operatorname{cl}(X)$ is the biggest fully connected subset containing $X$.

Proof: Consider an arbitrary fully connected subset $Y$ such that $X \subset Y$. Let us prove that $Y \subset \operatorname{cl}(X)$. Consider $x \in Y$ and an arbitrary $y \in X$. Since $Y$ is fully connected we have that $x \leftrightarrow y$. This means that $x \in \operatorname{cl}(X)$. Hence, we have proven that $Y \subset \operatorname{cl}(X)$.

\section{Appendix 4}

Proposition: Consider $x, y \in \mathcal{X}$ two episodes. For $c l(\{x\})$ and $\operatorname{cl}(\{y\})$ we have

$$
c l(\{x\})=\operatorname{cl}(\{y\}) \quad \text { or } \quad c l(\{x\}) \cap \operatorname{cl}(\{y\})=\emptyset
$$

Proof: Suppose that $\operatorname{cl}(\{x\}) \cap \operatorname{cl}(\{y\}) \neq \emptyset$, which means that there exists an episode $z \in \operatorname{cl}(\{x\})$ and $z \in \operatorname{cl}(\{y\})$. Hence, we have $z \leftrightarrow x$ and $z \leftrightarrow y$. From this it follows that $x \leftrightarrow y$. But then $x \in \operatorname{cl}(\{y\})$, and hence, $\{x\} \subset \operatorname{cl}(\{y\})$. Applying (18) we get $\operatorname{cl}(\{x\}) \subset \operatorname{cl}(\{y\})$. Analogously it can be shown that $\operatorname{cl}(\{y\}) \subset \operatorname{cl}(\{x\})$, and as a consequence $\operatorname{cl}(\{x\})=\operatorname{cl}(\{y\})$. This proves the proposition, namely that for the closure of two single episodes, the closure subsets are equal, or their intersection is empty.

\section{Appendix 5}

We now prove that $\mathcal{X}$ is equal to the disjoint union of fully connected subsets, such that each of the subsets is the closure of a single episode.

Proposition: We have

$$
\mathcal{X}=\bigcup_{i \in I} X_{i} \quad X_{i} \text { fully connected } \quad X_{j} \cap X_{k}=\emptyset \text { for } j \neq k
$$

such that for each $X_{i}$ there exists a single episode $x_{i} \in \mathcal{X}$, such that $X_{i}=$ $\operatorname{cl}\left(\left\{x_{i}\right\}\right)$.

Proof: From set theory it follows that $\mathcal{X}=\cup_{x \in \mathcal{X}}\{x\}$, and since for each $x \in \mathcal{X}$ it is the case that $\{x\} \subset \operatorname{cl}(\{x\})$ then also $\mathcal{X}=\cup_{x \in \mathcal{X}} c l(\{x\})$. Consider the set the set $\{\operatorname{cl}(\{x\}) \mid x \in \mathcal{X}\}$. From the above proposition it follows that if $\operatorname{cl}(\{y\})$ and $c l(\{z\})$ are two elements of this set, $\operatorname{cl}(\{y\})=\operatorname{cl}(\{z\})$ or $\operatorname{cl}(\{y\}) \cap \operatorname{cl}(\{z\})=\emptyset$. This proves the proposition. 


\section{References}

Aerts, D. (1982). Description of many physical entities Without the paradoxes encountered in quantum mechanics. Foundations of Physics, 12, 11311170.

Aerts, D. (1983). Classical theories and non classical theories as a special case of a more general theory, Journal of Mathematical Physics, 24, 2441-2453.

Aerts, D. (2007). General quantum modeling of combining concepts: A quantum field model in Fock space. Archive address and link: http://arxiv.org/abs/0705.1740.

Aerts, D. (2009). Quantum structure in cognition. Journal of Mathematical Psychology (submitted).

Aerts, D., Broekaert, J. and Smets, S. (1999a). The liar paradox in a quantum mechanical perspective. Foundations of Science, 4, pp. 115-132.

Aerts, D., Broekaert, J., Smets, S. (1999b). A quantum structure description of the liar paradox. International Journal of Theoretical Physics, 38, pp. 3231-3239.

Aerts, D. \& Gabora, L. (2005a). A theory of concepts and their combinations I: The structure of the sets of contexts and properties. Kybernetes, $34(1 \& 2)$, 167-191.

Aerts, D. \& Gabora, L. (2005b). A theory of concepts and their combinations II: A Hilbert space representation. Kybernetes, 34(1\&2), 192-221.

Aiello, L. \& Dunbar, R. (1993). Neocortex size, group size, and the evolution of language. Current Anthropology, 34, 184-193.

Ashby, F. G., \& Ell, S. W. (2002). Single versus multiple systems of learning and memory. In J. Wixted \& H. Pashler (Eds.) Stevens? handbook of experimental psychology: Volume 4 Methodology in experimental psychology. New York: Wiley.

Barkow, J., Cosmides, L., \& Tooby, J. (1992). The adapted mind. New York: Oxford University Press.

Bar-Yosef, O. (1994). The contribution of southwest Asia to the study of the origin of modern humans. In M. Nitecki \& D. Nitecki (Eds.) Origins of anatomically modern humans, Plenum Press. Bouissac, P. (1998). Converging parallels: Semiotics and psychology in evolutionary perspective. Theory and Psychology, 8(6), 731-753.

Beltrametti, E. \& Cassinelli, G. (1981). The logic of quantum mechanics. Boston: Addison-Wesley.

Bickerton, D. (1990). Language and species. Chicago: University of Chicago Press. 
Barsalou, L.W. (1982). Context-independent and context-dependent information in concepts. Memory $\&$ Cognition, 10, 82-93.

Bruza, P. \& Cole, R. (2005). Quantum logic of semantic space: An exploratory investigation of context effects in practical reasoning. In S. Artemov, $\mathrm{H}$. Barringer, A. S. d'Avila Garcez, L. C. Lamb and J. Woods (Eds.) We Will Show Them: Essays in Honour of Dov Gabbay. College Publications, 1, pp. 339?361. Bruza, P. Kitto, K. Nelson, D. \& McEvoy, C. (in press). Is there something quantum-like about the human mental lexicon? Journal of Mathematical Psychology.

Busemeyer, J. R., Wang, Z., \& Townsend, J. T. (2006). Quantum dynamics of human decision making. Journal of Mathematical Psychology, 50, 220242 .

Busemeyer, J. R. , Matthew, M., \& Wang, Z. (2006) A Quantum Information Processing Theory Explanation of Disjunction Effects. Proceedings of the Cognitive Science Society.

Busemeyer, J. R. \& Wang, Z. (2007). Quantum Information Processing Explanation for Interactions between Inferences and Decisions. Proceedings of the AAAI Spring Symposium on Quantum Interaction (pp. 91-97). Stanford University, March 26-28.

Carey, S. (1985). Conceptual change in childhood. Cambridge: MIT Press.

Churchland, P. S. \& Sejnowski, T. (1992). The computational brain. Cambridge MA: MIT Press.

Corballis, M. C. (1991). The lopsided ape: Evolution of the generative mind. Cambridge: Cambridge University Press.

Davis, J., \& Rovee-Collier, C. (1983). Alleviated forgetting of a learned contingency in 8-week-old infants. Developmental Psychology, 19, 353-365.

Dawkins, R. (1976). The selfish gene. Oxford: Oxford University Press.

Deacon, T. (1997). The symbolic species. New York: Norton. [p. 751]

Dennett, D. (1978). Brainstorms. Cambridge, MA: MIT Press.

Dikranjan, D., Giuli, E. \& Tozzi, A. (1988). Topological categories and closure operators. Questiones Mathematics, 11(3), 323-337.

Donald, M. (1991) Origins of the modern mind. Cambridge MA: Harvard University Press.

Edelman, G. E. (1993). Bright Air, Brilliant Fire: On the Matter of Mind. New York: Basic Books. 
Erdos, P. \& Renyi, A. (1959). On the random graphs. 1(6), Institute of Mathematics, University of Debrecenians, Debrecar, Hungary.

Erdos, P. \& Renyi, A. (1960). On the evolution of random graphs. Institute of Mathematics, Hungarian Academy of Sciences Publication number 5.

Fauconnier, G. \& Turner, M. (2002). The way we think: Conceptual blending and the mind's hidden complexities. New York: Basic Books.

Gabora, L. (1998). A tentative scenario for the origin of culture. Psycoloquy, $9(67)$.

Gabora, L. (1999). Weaving, bending, patching, mending the fabric of reality: A cognitive science perspective on worldview inconsistency. Foundations of Science, 3(2), 395-428.

Gabora, L. (2000). Conceptual closure: Weaving memories into an interconnected worldview. In G. Van de Vijver and J. Chandler (Eds.) Closure: Emergent Organizations and their Dynamics. Annals of the New York Academy of Sciences 901, 42-53.

Gabora, L. (2002a). The beer can theory of creativity. In P. Bentley \& D. Corne (Eds.) Creative Evolutionary Systems, (pp. 147-161). San Francisco CA: Morgan Kauffman.

Gabora, L. (2002b). Cognitive mechanisms underlying the creative process. In T. Hewett and T. Kavanagh (Eds.) Proceedings of the Fourth International Conference on Creativity and Cognition, (pp. 126-133). October 13-16, Loughborough University, UK.

Gabora, L. (2003). Contextual focus: A cognitive explanation for the cultural transition of the Middle/Upper Paleolithic. In (R. Alterman \& D. Hirsch, Eds.) Proceedings of the 25th Annual Meeting of the Cognitive Science Society, Boston MA, 31 July-2 August. Lawrence Erlbaum Associates.

Gabora, L. (2004). Ideas are not replicators but interrelated networks of them are. Biology and Philosophy, 19(1), 127-143.

Gabora, L. (2005). Creative thought as a non-Darwinian evolutionary process. Journal of Creative Behavior, 39(4), 65-87.

Gabora, L. (2006). Self-other organization: Why early life did not evolve through natural selection. Journal of Theoretical Biology, 241 (3), 443450 .

Gabora, L. (2008). The cultural evolution of socially situated cognition. Cognitive Systems Research, 9(1-2), 104-113.

Gabora, L. \& Aerts, D. (2002). Contextualizing concepts using a mathematical generalization of the quantum formalism. Journal of Experimental and Theoretical Artificial Intelligence, 14(4), 327-358. 
Gabora, L. \& Aerts, D. (2005). Evolution as context-driven actualization of potential: Toward an interdisciplinary theory of change of state. Interdisciplinary Science Reviews, 30(1), 69-88.

Gabora, L., Rosch, E., \& Aerts, D. (2008). Toward an ecological theory of concepts. Ecological Psychology, 20(1), 84-116.

Gärdenfors, P. (2000). Conceptual Spaces: The geometry of thought. Cambridge, MA: MIT press.

Gelman, R. (1993). A rational-constructivist account of early learning about numbers and objects. In D. Medin (Ed.) Learning and motivation, (pp. 61-96). New York: Academic Press.

Hampton, S. J. (2004). Domina mismatches, scruffy engineering, exaptations and spandrels. Theory and Psychology 14(2), 147-166.

Hancock, P. J. B., Smith, L. S. \& Phillips, W. A. (1991). A biologically supported error-correcting learning rule. Neural Computation, 3(2), 201212 .

Holden, S.B. \& Niranjan, M. (1997). Average-case learning curves for radial basis function networks. Neural Computation, 9(2), 441-460.

Johnson-Laird, P. N. (1983). Mental models. Cambridge MA: Harvard University Press.

Kanerva, P. (1988). Sparse, distributed memory. Cambridge MA: MIT Press.

Karmiloff-Smith, A. (1990). Constraints on representational change: Evidence from children?s drawing. Cognition, 34, 57-83.

Karmiloff-Smith, A. (1992). Beyond modularity: A developmental perspective on cognitive science. Cambridge MA: MIT Press.

Kauffman, S. (1993). Origins of order. Oxford: Oxford University Press.

Keil, F. C. (1995). The growth of causal understandings of natural kinds. In S. Sperber, D. Premack, \& A. Premack (Eds.) Causal cognition: A multidisciplinary debate, (pp. 234-262). Cambridge: Oxford University Press.

Klein, R. (1989). Biological and behavioral perspectives on modern human origins in South Africa. In P. Mellars \& C. Stringe (Eds.) The human revolution. Edinburgh: Edinburgh University Press.

Langton, C. G. (1992) Life at the edge of chaos. In (C. G. Langton, C. Taylor, J. D. Farmer \& S. Rasmussen, eds.) Artificial life II. Boston: AddisonWesley. 
Leakey, R. (1984). The origins of humankind. New York: Science Masters Basic Books.

Leslie, A. (2000). Theory of mind as a mechanism of selective attention. Rutgers University Tech Report 061.

Lieberman, P. (1991). Uniquely human: The evolution of speech, thought, and selfless behavior. Boston: Harvard University Press.

Lu, Y.W., Sundararajan, N. \& Saratchandran, P. (1997). A sequential learning scheme for function approximation using minimal radial basis function neural networks. Neural Computation, 9(2), 461-478.

Maturana, H. \& Varela, F. (1973/1980). Autopoeisis and cognition: The realization of the living. Boston: Reidel.

Matzel, L., Collin, C., \& Alkon, D. (1992). Biophysical and behavioral correlates of memory storage: Degradation and reactivation. Behavioral Neuroscience, 106, 954-963.

McBrearty, S. \& Brooks, A. S. (2000). The revolution that wasn?t: A new interpretation of the origin of modern human behavior. Journal of Human Evolution 39(5), 453-563.

Mednick, S. (1962). The associative basis of the creative process. Psychological Review, 69, 220?232.

Mellars, P. (1973). The character of the middle-upper transition in south-west France. In C. Renfrew (Ed.) The explanation of culture change. London: Duckworth.

Mellars, P. (1989a). Technological changes in the Middle-Upper Paleolithic transition: Economic, social, and cognitive perspectives. In P. Mellars \& C. Stringer (Eds.) The human revolution. Edinburgh: Edinburgh University Press.

Mellars, P. (1989b). Major issues in the emergence of modern humans. Current Anthropology, 30, 349-385.

Mikkulainen (1997). Neural network perspectives on cognition and adaptive robotics. In (A Brown, Ed.) Natural language processing with subsymbolic neural networks. Bristol UK, Philadelphia: Institute of Physics Press.

Mithen, S. (1996). The prehistory of the mind: A search for the origins of art, science, and religion. London: Thames \& Hudson.

Mithen, S. (1998). A creative explosion? Theory of mind, language, and the disembodied mind of the Upper Paleolithic. In S. Mithen (Ed.) Creativity in human evolution and prehistory. New York: Routledge. 
Morowitz, H. J. (2002). The Emergence of Everything: How the World Became Complex. Oxford University Press, New York.

Neisser, U. (1963). The multiplicity of thought. British Journal of Psychology, $54,1-14$.

Nelson, D. L. \& McEvoy, C. L. (2007). Activation at a distance is not spooky anymore: The conjunction of entangled associative structures and context. Proceedings of the AAAI Spring Symposium on Quantum Interaction, Stanford University, March 26-28.

Nelson, D. L., McEvoy, C. L. \& Pointer, L. (2003). Spreading activation or spooky action at a distance. Journal of Experimental Psychology: Learning, Memory, and Cognition 29, 42?51.

Osherson , D. N. \& Smith, E. E. (1981). On the adequacy of prototype theory as a theory of concepts. Cognition, 9, 35-58.

Piaget 1926). The language and thought of the child. Hillsdale, NJ: Lawrence Erlbaum.

Piron, C. (1976). Foundations of Quantum Physics. Reading, MA: W.A. Benjamin.

Rosch (1978). Principles of categorization. In Rosch, E. \& Lloyd, B. (Eds.) Cognition and categorization. Hillsdale, NJ: Lawrence Erlbaum.

Rovee-Collier, C., Hartshorn, K., \& DiRubbo, M. (1999). Long-term maintenance of infant memory. Developmental Psychobiology, 35, 91-102.

Ruff, C., Trinkaus, E. \& Holliday, T. (1997). Body mass and encephalization in Pleistocene Homo. Nature, 387, 173-176.

Sloman, S. (1996). The empirical case for two systems of Reasoning. Psychological Bulletin, 9(1), 3-22.

Smolensky, P. (1988). On the proper treatment of connectionism. Behavioral and Brain Sciences, 11, 1?43.

Soffer, O. (1994). Ancestral lifeways in Eurasia-The Middle and Upper Paleolithic records. In M. Nitecki \& D. Nitecki, (Eds.) Origins of anatomically modern humans. New York: Plenum Press.

Spelke, E. S. (2000). Core knowledge. American Psychologist, 55(11), 12331243.

Spoor, F. Leakey, M. G., Gathogo, P. N., Brown, F. H., Anton, S. C., McDougall, I., Kiarie, C., Manthi, F. K. and Leakey, L. N. (2007). Implications of new early homo fossils from ileret, east of lake turkana, kenya. Nature, 448, 688-691. 
Stringer, C. \& Gamble, C. (1993). In search of the Neanderthals. London: Thames \& Hudson.

Thompson, E.(2007). Mind in Life - Biology, Phenomenology and the Sciences of Mind. Cambridge MA: Harvard University Press.

Vetsigian, K., Woese, C., \& Goldenfeld, N. (2006). Collective evolution and the genetic code. Proceedings of the New York Academy of Science USA, 103, 10696?10701.

W?chtersh?user, G. (1992). Groundwork for an evolutionary biochemistry: the iron-sulfur world. Prog. Biophys. Molec. Biol. 58, 85-201.

Weber, B. H. (1998). Emergence of life and biological selection from the perspective of complex systems dynamics. In: G. van de Vijver, S. N. Salthe \& M. Delpos (Eds.) Evolutionary Systems: Biological and Epistemological Perspectives on Selection and Self-Organization. Kluwer, Dordrecht.

Weber, B. H. (2000). Closure in the emergence and evolution of life: Multiple discourses or one? In: J. L. R. Chandler \& G. Van de Vijver (Eds.) Closure: Emergent Organizations and their Dynamics, Annals of the New York Academy of Sciences 901, pp. 132-138.

Weber, B.H., \& Depew, J.D. (1996). Natural selection and self-organization. Biology and Philosophy, 11(1), 33-65.

White, R. (1982). Rethinking the Middle/Upper Paleolithic transition. Current Anthropology, 23, 169-189.

White, R. (1993). Technological and social dimensions of 'Aurignacian-age' body ornaments across Europe. In H. Knecht, A. Pike-Tay, \& R. White (Eds.) Before Lascaux: The complex record of the Early Upper Paleolithc. New York: CRC Press.

Widdows, D. S. (2003). Orthogonal negation in vector spaces for modeling word meanings and document retrieval. In Proceedings of the 41st Annual Meeting of the Association for Computational Linguistics (p. 136-143), Sapporo Japan, July 7-12.

Widdows, D. \& Peters, S. (2003). Word vectors and quantum logic: experiments with negation and disjunction. In Eighth Meeting of the Association for the Mathematics of Language (p. 141-154), Bloomington IN: Association for Computational Linguistics.

Williams, R. J. P. \& Frausto da Silva, J. J. R. (1999). Bringing Chemistry to Life: From Matter to Man. Oxford University Press, Oxford.

Williams, R. J. P. \& Frausto da Silva, J. J. R. (2002). The systems approach to evolution. Biochem. Biophys. Res. Comm. 297, 689-699. 
Williams, R. J. P. \&Frausto da Silva, J. J. R. (2003). Evolution was chemically constrained. J. Theor. Biol. 220, 323-343.

Willshaw, D. J. \& Dayan, P. (1990). Optimal plasticity from matrix memory: What goes up must come down. Journal of Neural Computation, 2, 85-93.

Woese, C.R. (2002). On the evolution of cells. Proceedings of the National Academy of Sciences, 99(13), 8742-8747. 